hep-th/0602277

HIP-2006-10/TH

TIT/HEP-551

Feb., 2006

\title{
Hyper-Kähler Sigma Models on (Co)tangent Bundles with $S O(n)$ Isometry
}

\author{
Masato $\operatorname{Arai}^{a *}$ and Muneto Nitta $^{b \dagger}$ \\ ${ }^{a}$ High Energy Physics Division, Department of Physical Sciences, University of Helsinki \\ and Helsinki Institute of Physics, P.O.Box 64, FIN-00014, Finland \\ ${ }^{b}$ Department of Physics, Tokyo Institute of Technology \\ Tokyo 152-8551, Japan
}

\begin{abstract}
We construct $\mathcal{N}=2$ supersymmetric nonlinear sigma models whose target spaces are tangent as well as cotangent bundles over the quadric surface $Q^{n-2}=$ $S O(n) /[S O(n-2) \times U(1)]$. We use the projective superspace framework, which is an off-shell formalism of $\mathcal{N}=2$ supersymmetry.
\end{abstract}

\footnotetext{
${ }^{*}$ E-mail: masato.arai@helsinki.fi

†E-mail: nitta@th.phys.titech.ac.jp
} 


\section{Introduction}

Hyper-Kähler manifolds provide a fruitful relation between physics and mathematics. One of ingredients to study them may be solitons. For instance it is well-known that the moduli space for Yang-Mills (YM) instantons on $\mathbf{R}^{4}$ [1] and one of Bogomol'nyi-Prasad-Sommerfield (BPS) monopoles [2, 3] are both hyper-Kähler. Gravitational instantons [4, 5] and the moduli space of YM instantons on gravitational instantons [6] are also hyper-Kähler. More direct connection related to these would be given by supersymmetric nonlinear sigma models with eight supercharges (like $\mathcal{N}=2$ supersymmetry in $d=4$ and $\mathcal{N}=(4,4)$ supersymmetry in $d=2$ ): scalar fields in these models belong to hypermultiplets, parametrizing target spaces which must be hyper-Kähler from the requirement of $\mathcal{N}=2$ supersymmetry [7. Conversely there exists the unique (massless) nonlinear sigma model for arbitrary hyper-Kähler manifold. Hyper-Kähler structure on soliton moduli spaces can be understood in terms of nonlinear sigma models as follows. Instantons and BPS monopoles can be naturally embedded into supersymmetric gauge theories with sixteen supercharges, and they preserve/break half of supersymmetry. Their dynamics can be described by nonlinear sigma models with eight supersymmetry preserved by them. The hyper-Kähler quotient was discovered in the context of nonlinear sigma models [8, 9]. Since then it has become an important tool: YM instantons, gravitational instantons and YM instantons on gravitational instantons can be obtained by certain hyper-Kähler quotients [1, 5, 6]. Hyper-Kähler sigma models also give low energy effective action on the Higgs branch of vacua in $\mathcal{N}=2$ supersymmetric gauge theories [10, 11, where target space is obtained as the hyper-Kähler quotient.

Construction of explicit metrics on hyper-Kähler manifolds is an important problem. Compact hyper-Kähler manifolds are difficult to construct whereas known ones are all non-compact. In general an isometry of manifolds often restricts the form of their metrics. An important class of hyper-Kähler manifolds is given by toric hyper-Kähler (hypertoric) manifolds [8, 12, 13, 14, 15], namely $4 n$ dimensional hyper-Kähler manifolds admitting mutually commuting $n$ tri-holomorphic isometries. This class of manifolds was firstly found in construction of the general action of $\mathcal{N}=2$ supersymmetric tensor multiplets [8, which can be dualized by the Legendre transformation to hypermultiplets with toric hyper-Kähler manifolds. Thus $\mathcal{N}=2$ supersymmetric models are obviously useful to study hyper-Kähler manifolds. Any toric hyper-Kähler manifold (with dimension $4 n$ ) can be obtained as hyper-Kähler quotient of flat space $\mathbf{H}^{n+m}$ by $U(1)^{m}[14$. HyperKähler manifolds which are not toric were studied with hyper-Kähler quotient by a product of gauge group $\Pi_{i=1}^{k} U\left(N_{i}\right)$ in Ref. [16].

An isometry of non-Abelian group $G$ is further restrictive for metrics of manifolds. Homogeneous Kähler manifolds $G / H$ were completely classified [17, 18] and their Kähler potentials were systematically constructed [19]. Hyper-Kähler manifolds cannot become homogeneous, so we may consider in a slightly different way. Let us remember that homogeneous Kähler manifolds can be formulated as co-adjoint orbits of Lie algebra $G$ with the so-called Kirillov-Kostant-Souriau

symplectic structure [20]. Then, co-adjoint orbits of complex Lie algebra $G^{\mathbf{C}}$ become cotangent bundles $G^{\mathbf{C}} / H^{\mathbf{C}} \simeq T^{*}(G / H)$ over homogeneous Kähler manifolds $G / H$, and they are known 
to admit hyper-Kähler metrics [21. ${ }^{1}$ More explicit analysis was performed for cotangent bundle over Hermitian symmetric spaces [25]. Later it was shown that cotangent bundles over any Kähler manifolds $M$ (without any isometry) admit hyper-Kähler metrics at least in neighbour of $M$ [26, 27].

When one would like to construct arbitrary hyper-Kähler manifold, fully off-shell $\mathcal{N}=2$ supersymmetric formalisms should be useful. The harmonic superspace provides such a fully off-shell $\mathcal{N}=2$ superspace [28, 29]. It can provide the most general action for hypermultiplets which induces the most general hyper-Kähler manifolds if one can eliminate infinite number of auxiliary fields. The projective superspace [30] 37] is another fully off-shell $\mathcal{N}=2$ superspace. Its equivalence to harmonic superspace was discussed 38. Recently the projective superspace in five- and six-dimensions has also been studied [39, 40, 41. In the six-dimensional case, the projective superspace was first introduced in [42] to construct self-coupling of $\mathcal{N}=(1,0)$ tensor multiplets.

It was shown by Gates and Kuzenko [43, 44 that the particular multiplets in the projective superspace, called the polar multiplets, are suitable to describe $\mathcal{N}=2$ supersymmetric nonlinear sigma models on tangent (but not cotangent) bundles $T M$ over Kähler manifolds $M .^{2}$ The polar multiplets $\Upsilon^{i}$ contain chiral superfields $\Phi^{i}$ and complex linear (nonminimal) superfields $\Sigma^{i} 46$ ] in terms of $\mathcal{N}=1$ superfields, where $\Phi^{i}$ parametrize the base Kähler manifold $M$ and $\Sigma^{i}$ are regarded as components of a tangent vector on $M$. Complex linear superfields $\Sigma^{i}$ can be dualized by the Legendre transformation to chiral superfields $\psi_{i}$ which can be regarded as components of a cotangent vector. Then, the nonlinear sigma models on cotangent bundles $T^{*} M$ in terms of solely chiral superfields $\left(\Phi^{i}, \psi_{i}\right)$ are obtained. Once the Kähler potential $K\left(\Phi, \Phi^{\dagger}\right)$ of $\mathcal{N}=1$ supersymmetric model on any Kähler manifold $M$ is given, one can easily obtain its $\mathcal{N}=2$ supersymmetric extension on (co)tangent bundle $T^{(*)} M$ with replacing chiral superfields $\Phi^{i}$ by polar multiplets $\Upsilon^{i}$. This is nicely conforming to the mathematical result [26, 27]. ${ }^{3}$ However, the main problem to obtain explicit action in terms of component fields (or $\mathcal{N}=1$ superfields) is that one has to eliminate infinite number of auxiliary $\mathcal{N}=1$ superfields contained in the polar multiplets. The authors in [43, 44] explicitly constructed nonlinear sigma models on tangent and cotangent bundles over the complex projective space $\mathbf{C} P^{n-1}=S U(n) /[S U(n-1) \times U(1)]$, which is one of the Hermitian symmetric spaces, by eliminating auxiliary fields with the help of the isometry $S U(n)$ on $\mathbf{C} P^{n-1}$. The cotangent bundle action recovers the $T^{*} \mathbf{C} P^{n-1}$ sigma model constructed by the hyper-Kahler quotients [4]. The purpose of the present paper is to construct $\mathcal{N}=2$ supersymmetric nonlinear sigma models on (co)tangent bundle on another Hermitian symmetric space, the so-called quadric surface $Q^{n-2}=S O(n) /[S O(n-2) \times U(1)]$, with following their work.

This paper is organized as follows. We give a review of the Kähler quotient construction of $Q^{n}$ [48, 49] in the rest of introduction. In section 2, we give a brief review of the projective superspace. In section 3, we review how to construct nonlinear sigma models with tangent and

\footnotetext{
${ }^{1}$ Stenzel constructed the Ricci-flat Kähler metric on complexification of Riemann symmetric spaces $G^{\mathbf{C}} / H^{\mathbf{C}} \simeq$ $T^{*}(G / H)$ [22]. See [23, 24] for explicit metric in the case of $S^{N-1} \simeq S O(N) / S O(N-1)$.

${ }^{2}$ They also studied hyper-Kähler manifolds by using c-map in the projective superspace framework 45 .

${ }^{3}$ The work of Gates and Kuzenko was done independently from [26, 27]. In fact it was earlier than [27].
} 
cotangent bundles over the projective space $\mathbf{C} P^{n-1}$. We consider a sigma model with tangent bundle $T Q^{n-2}$ in section 4 . In subsection 4.1 , by using the isomorphism $Q^{2} \simeq \mathbf{C} P^{1} \times \mathbf{C} P^{1}$, we construct the sigma model with tangent bundle $T Q^{2}$. In subsection 4.2 , we solve the equations of motion for auxiliary fields and derive the $T Q^{n-2}$ action. In section 5 , we derive the nonlinear sigma action with cotangent bundle $T^{*} Q^{2}$ via the Legendre transformation. Further we propose the the cotangent bundle action for $T^{*} Q^{n-2}$. Section 6 is devoted to discussion. In Appendix A, we review an another method to eliminate infinite set of auxiliary fields based on the duality between polar and $O(2)$ multiplet with some examples. In Appendix B, we show the detailed derivation of identities between metric and Riemann tensor. In Appendix C, we derive the sigma model with cotangent bundle $T^{*} Q^{2}$ with the isomorphism $T^{*} Q^{2} \simeq T^{*} \mathbf{C} P^{1} \times T^{*} \mathbf{C} P^{1}$. We discuss the $n=3$ case in $T^{(*)} Q^{n-2}$ sigma model, and show that the solution for $\Upsilon$ and the (co)tangent bundle action is $T^{(*)} \mathbf{C} P^{1}$ 's one in Appendix D.

Before closing introduction we review how to construct nonlinear sigma models on the quadric surface $Q^{n-2}$ in terms of the $\mathcal{N}=1$ superfields [48, 49]. Let $\phi^{i}(x, \theta, \bar{\theta})(i=1, \cdots, n)$ be chiral superfields, $\bar{D}_{\dot{\alpha}} \phi^{i}=0$ belonging to the vector representation of $S O(n)$. Introducing an auxiliary vector superfield $V(x, \theta, \bar{\theta})\left(=V^{\dagger}\right)$ and an auxiliary chiral superfield $\sigma(x, \theta, \bar{\theta})\left(\bar{D}_{\dot{\alpha}} \sigma=0\right)$, being a singlet representation of $S O(n)$, the Lagrangian can be written as

$$
\mathcal{L}=\int d^{4} \theta\left(\phi^{i \dagger} \phi^{i} e^{V}-r^{2} V\right)+\left(\int d^{2} \theta \sigma \phi^{i} \phi^{i}+\text { c.c. }\right)
$$

with summation over repeated index $i$ implied, and $r^{2}$ a real positive constant called FayetIliopoulos parameter. This Lagrangian possesses gauge invariance

$$
V \rightarrow V-\Lambda-\Lambda^{\dagger}, \quad \phi^{i} \rightarrow e^{\Lambda} \phi^{i}, \quad \sigma \rightarrow e^{-2 \Lambda} \sigma
$$

with arbitrary chiral superfield $\Lambda(x, \theta, \bar{\theta})$. Equation of motion of $V$ read $\phi^{i \dagger} \phi^{i} e^{V}-r^{2}=0$, which can be solved as $V=-\log \left(\phi^{i \dagger} \phi^{i} / r^{2}\right)$. When the superpotential is absent in the Lagrangian (1.1), we obtain the Kähler potential of $\mathbf{C} P^{n-1}$ by substituting the solution back into the Kähler potential of (1.1) as

$$
K=r^{2} \log \left(1+\frac{\left|\Phi^{i}\right|^{2}}{r^{2}}\right)
$$

with a gauge fixing $\vec{\phi}=\left(\Phi^{i}, r\right)(i=1, \cdots, n-1)$. But now there exists the superpotential in the Lagrangian (1.1). Decomposing $\phi^{i}$ in the representation of the $S O(n-2) \times U(1)$ group of $S O(n)$ as $\phi^{i}=\left(x, y^{j}, z\right)(j=1, \cdots, n-2)$, the $S O(n)$ transformation law is given by [48]

$$
\delta \phi^{i}=\left(\begin{array}{ccc}
i \theta & \bar{\epsilon}^{\bar{j}} & 0 \\
-\epsilon^{i} & \theta^{i j} & -\bar{\epsilon}^{i} \\
0 & \epsilon^{j} & -i \theta
\end{array}\right)\left(\begin{array}{c}
x \\
y^{j} \\
z
\end{array}\right),
$$

where $\theta^{i j}=\frac{i}{2} \theta^{k l}\left(T^{k l}\right)^{i j}$ and $\left(T^{i j}\right)^{k l}=\frac{1}{i}\left(\delta^{i k} \delta^{j l}-\delta^{k j} \delta^{i l}\right)$. We take the rank-2 invariant tensor as

$$
J=\left(\begin{array}{ccc}
0 & \mathbf{0} & 1 \\
\mathbf{0} & \mathbf{1}_{n-2} & \mathbf{0} \\
1 & \mathbf{0} & 0
\end{array}\right) .
$$


The equation of motion of $\sigma$ gives the constraint

$$
\vec{\phi}^{2}=\phi^{T} J \phi=2 x z+y^{2}=0 .
$$

This can be solved to give

$$
\vec{\phi}=\left(\begin{array}{c}
x \\
y^{j} \\
-\frac{y^{2}}{2 x}
\end{array}\right)
$$

With a gauge fixing $x=r, \vec{\phi}^{T}=\left(r, \Phi^{i},-\frac{1}{2 r} \Phi^{2}\right)$, we obtain the Kähler potential of the quadric surface [50, 48, 49, 51], given by

$$
K\left(\Phi^{i}, \bar{\Phi}^{\bar{j}}\right)=r^{2} \ln \left(1+\frac{\left|\Phi^{i}\right|^{2}}{r^{2}}+\frac{\left(\Phi^{i}\right)^{2}\left(\bar{\Phi}^{\bar{j}}\right)^{2}}{4 r^{4}}\right)
$$

The Kähler metric can be calculated to give

$$
g_{i \bar{j}}=\frac{\partial^{2} K}{\partial \Phi^{i} \partial \bar{\Phi}^{\bar{j}}}=\frac{\delta_{i \bar{j}}}{1+\frac{\left|\Phi^{k}\right|^{2}}{r^{2}}+\frac{\left(\Phi^{l}\right)^{2}\left(\bar{\Phi}^{\bar{m}}\right)^{2}}{4 r^{4}}}+\frac{\frac{\Phi^{i} \bar{\Phi}^{\bar{j}}-\Phi^{j} \bar{\Phi}^{\bar{i}}}{r^{2}}+\frac{2 \Phi^{i} \bar{\Phi}^{\bar{j}}\left|\Phi^{k}\right|^{2}-\Phi^{i} \Phi^{j}\left(\bar{\Phi}^{k}\right)^{2}-\bar{\Phi}^{i} \bar{\Phi}^{\bar{j}}\left(\Phi^{k}\right)^{2}}{2 r^{4}}}{\left(1+\frac{\left|\Phi^{l}\right|^{2}}{r^{2}}+\frac{\left(\Phi^{m}\right)^{2}\left(\bar{\Phi}^{\bar{n}}\right)^{2}}{4 r^{4}}\right)^{2}} .
$$

\section{Projective Superspace}

The projective superspace [30] 37] consists of a complex projective coordinate $\zeta$, which is an inhomogeneous coordinate of $\mathbf{C} P^{1}$, in addition to $\mathcal{N}=2$ global superspace $\mathbf{R}^{4 \mid 8}$ parameterized by

$$
z^{M}=\left(x^{\mu}, \theta^{i \alpha}, \bar{\theta}_{\dot{\alpha}}^{i}\right), \quad \overline{\theta_{i}^{\alpha}}=\bar{\theta}^{\dot{\alpha} i}, \quad i=1,2
$$

where the index $i$ labels the fundamental representation of the automorphism group $S U(2)_{R}$. Superfields $\Upsilon$ on the projective superspace are functions of this projective superspace with the constraints

$$
\nabla_{\alpha} \Upsilon(z, \zeta)=\bar{\nabla}_{\dot{\alpha}} \Upsilon(z, \zeta)=0
$$

where $\nabla_{\alpha}$ and $\bar{\nabla}_{\dot{\alpha}}$ are linear combination of $\mathcal{N}=2$ supercovariant derivatives in four dimensions, given by

$$
\nabla_{\alpha}(\zeta)=D_{1 \alpha}+\zeta D_{2 \alpha}, \quad \bar{\nabla}_{\dot{\alpha}}(\zeta)=\bar{D}_{\dot{\alpha}}^{2}-\zeta \bar{D}_{\dot{\alpha}}^{1}
$$

Here the supercovariant derivatives satisfy the following algebra ${ }^{4}$

$$
\left\{D_{i \alpha}, D_{j \beta}\right\}=\left\{\bar{D}_{i \dot{\alpha}}, \bar{D}_{j \dot{\beta}}\right\}=0, \quad\left\{D_{i \alpha}, \bar{D}_{\dot{\beta}}^{j}\right\}=-2 i \delta_{i}^{j} \partial_{\alpha \dot{\beta}}
$$

\footnotetext{
${ }^{4}$ We take the normalization as $D^{2}=\frac{1}{4} D^{\alpha} D_{\alpha}$.
} 
Notice that $\bar{\nabla}_{\dot{\alpha}}$ is the conjugate of $\nabla_{\alpha}$ under the composition of complex conjugation with the antipodal map on the Riemann sphere, $\bar{\zeta} \rightarrow-1 / \zeta$, and multiplication by an appropriate factor. For example,

$$
\bar{\nabla}_{\dot{\alpha}}(\zeta)=(-\zeta)\left(\nabla_{\alpha}\right)^{*}\left(-\frac{1}{\zeta}\right)
$$

In the following, all conjugate of fields and operators in projective superspace are defined in this sense.

The constraints (2.2) for superfields are analogous to one for a chiral superfield in $\mathcal{N}=1$ superspace formalism where the chiral subspace is defined. The constraints (2.2) define a subspace of the full $\mathcal{N}=2$ superspace (2.1). Since a function $K(\Upsilon, \bar{\Upsilon})$ of superfields is independent of some (a half) of the Grassmann coordinates of $\mathcal{N}=2$ superspace by definition (2.2), its integration over the orthogonal operators for (2.3).

$$
\Delta_{\alpha}=\zeta^{-1} D_{1 \alpha}-D_{2 \alpha}, \quad \bar{\Delta}_{\dot{\alpha}}=\zeta^{-1} \bar{D}_{\dot{\alpha}}^{2}+\bar{D}_{\dot{\alpha}}^{1}
$$

is invariant under $\mathcal{N}=2$ supersymmetry. This leads to the following $\mathcal{N}=2$ invariant action:

$$
S=\frac{1}{32 \pi i} \int d^{4} x \oint_{C} \zeta d \zeta \frac{\Delta^{2} \bar{\Delta}^{2}}{16} K(\Upsilon, \bar{\Upsilon}, \zeta)
$$

Here the integration contour $C$ in the $\zeta$-plane is supposed to be chosen to make the action (2.7) nontrivial (i.e. not equal to zero). In the following, we take the contour to surround the origin in the $\zeta$-plane.

The algebra for $\nabla, \bar{\nabla}, \Delta$ and $\bar{\Delta}$ is given by

$$
\begin{aligned}
\{\nabla, \nabla\}= & \{\nabla, \bar{\nabla}\}=\{\Delta, \Delta\}=\{\Delta, \bar{\Delta}\}=\{\nabla, \Delta\}=0, \\
& \left\{\nabla_{\alpha}, \bar{\Delta}_{\dot{\alpha}}\right\}=-\left\{\bar{\nabla}_{\dot{\alpha}}, \Delta_{\alpha}\right\}=4 i \partial_{\alpha \dot{\alpha}} .
\end{aligned}
$$

Using Eqs. (2.2), (2.8) and (2.9) with the identities

$$
\Delta_{\alpha}=\zeta^{-1}\left(2 D_{\alpha}-\nabla_{\alpha}\right), \quad \bar{\Delta}_{\dot{\alpha}}=2 \bar{D}_{\dot{\alpha}}+\zeta^{-1} \bar{\nabla}_{\dot{\alpha}},
$$

the manifestly $\mathcal{N}=2$ supersymmetric action (2.7) can be reduced to the action in terms of $\mathcal{N}=1$ superfields,

$$
S=\int d^{4} x \frac{1}{2 \pi i} \oint_{C} \frac{d \zeta}{\zeta} \frac{D^{2} \bar{D}^{2}}{16} K(\Upsilon|, \bar{\Upsilon}|, \zeta)=\int d^{8} z \frac{1}{2 \pi i} \oint_{C} \frac{d \zeta}{\zeta} K(\Upsilon|, \bar{\Upsilon}|, \zeta)
$$

with $\mathcal{N}=1$ superfield covariant derivative defined by $D_{\alpha} \equiv D_{1 \alpha}$, and $d^{8} z \equiv d^{4} x D^{2} \bar{D}^{2} / 16=$ $d^{4} x d^{2} \theta d^{2} \bar{\theta}$. Here $\Upsilon \mid$ indicates the $\theta^{2}$ and $\bar{\theta}^{2}$ independent part of a superfield $\Upsilon$. In the following, we will simply write it as $\Upsilon$.

The superfields obeying the constraints (2.2) are classified into (i) real/complex $O(k)$ multiplets 31], (ii) rational multiplets [32, and (iii) analytic multiplets [32. Furthermore analytic 
multiplets contain the so-called polar multiplets and the real tropical multiplets, which describe charged $\mathcal{N}=2$ hypermultiplets and vector multiplets, respectively [36, 37. ${ }^{5}$ In what follows, we focus on the polar multiplets to consider $\mathcal{N}=2$ supersymmetric nonlinear sigma models. The polar multiplets and their conjugation can be expanded in terms of $\zeta$ as

$$
\Upsilon(z, \zeta)=\sum_{n=0}^{\infty} \Upsilon_{n}(z) \zeta^{n}, \quad \bar{\Upsilon}(z, \zeta)=\sum_{n=0}^{\infty} \bar{\Upsilon}_{n}(z)\left(-\frac{1}{\zeta}\right)^{n}
$$

respectively. ${ }^{6}$ Here all $\Upsilon_{n}$ (and $\bar{\Upsilon}_{n}$ ) are $\mathcal{N}=1$ superfields: $\Upsilon_{0}$ is a chiral superfield, $\Upsilon_{1}$ a complex linear (or nonminimal) superfield [46], satisfying the $\mathcal{N}=1$ constraints

$$
\bar{D}_{\dot{\alpha}} \Upsilon_{0}=0, \quad \bar{D}^{2} \Upsilon_{1}=0
$$

respectively, due to the constraints (2.2). The rests of fields $\Upsilon_{2}, \Upsilon_{3}, \ldots$, are complex unconstrained superfields, which are always auxiliary once the action is given.

The free action obeying hermiticity and $\mathcal{N}=2$ supersymmetry is given by

$$
S_{\text {free }}=\int d^{8} z \oint_{C} \frac{d \zeta}{2 \pi i \zeta} \bar{\Upsilon} \Upsilon
$$

On the other hand, the action (2.11) with the polar multiplets $\Upsilon$ is the most general action for $\mathcal{N}=2$ supersymmetric nonlinear sigma models on the tangent (but not cotangent) bundles over Kähler manifolds. For convenience let us rewrite physical $\mathcal{N}=1$ superfields in the projective superfields $\Upsilon^{i}$ (with $i$ labelling projective superfields) as

$$
\left.\Phi^{i} \equiv \Upsilon^{i}(\zeta)\right|_{\zeta=0},\left.\quad \Sigma^{i} \equiv \frac{d \Upsilon^{i}(\zeta)}{d \zeta}\right|_{\zeta=0}
$$

Then, $\Phi^{i}$ and $\Sigma^{i}$ are regarded as coordinates of the base Kähler manifold and components of a tangent vector, respectively, as explained as follows. The action (2.11) respects all the geometric features which $\mathcal{N}=1$ supersymmetric nonlinear sigma model on Kähler manifolds possesses. For instance, the action of $\mathcal{N}=1$ supersymmetric nonlinear sigma model

$$
S=\int d^{8} z K\left(\Phi^{i}, \bar{\Phi}^{\bar{i}}\right)
$$

is invariant under the Kähler transformation

$$
K(\Phi, \bar{\Phi}) \rightarrow K(\Phi, \bar{\Phi})+(\Lambda(\Phi)+\bar{\Lambda}(\bar{\Phi})) .
$$

This invariance can be promoted to

$$
K(\Upsilon, \bar{\Upsilon}) \rightarrow K(\Upsilon, \bar{\Upsilon})+(\Lambda(\Upsilon)+\bar{\Lambda}(\bar{\Upsilon}))
$$

\footnotetext{
${ }^{5}$ Cutting off the power series in (2.12) at some finite $k(>2)$, one results in the complex $O(k)$ multiplet. The case $k=1$ corresponds to the on-shell hypermultiplet, while for $k=2$ we obtain two tensor multiplets.

${ }^{6}$ The projective superfields $\Upsilon$ and $\bar{\Upsilon}$ are called arctic and antarctic 36, 37, respectively.
} 
for the action (2.11). A holomorphic field redefinition $\Phi^{i} \rightarrow f^{i}\left(\Phi^{j}\right)$ of the chiral superfields in the action (2.16) gives a holomorphic coordinate transformation. This is promoted for the action (2.11) to

$$
\Upsilon^{i} \rightarrow f^{i}\left(\Upsilon^{j}\right)
$$

deducing the transformation laws of $\Phi^{i}$ and $\Sigma^{i}$ as holomorphic coordinates of the base Kähler manifold, $\Phi^{i} \rightarrow f^{i}\left(\Phi^{j}\right)$, and components of a holomorphic tangent vector, $\Sigma^{i} \rightarrow \frac{\partial f^{i}}{\partial \Phi^{j}}(\Phi) \Sigma^{j}$. Thus, the set of fields $\left(\Phi^{i}, \Sigma^{i}\right)$ parameterizes the tangent bundles of the Kähler manifolds. Note that the action (2.11) is invariant under the rigid $U(1)$ transformations

$$
\Upsilon(\zeta) \rightarrow \Upsilon\left(e^{i \alpha} \zeta\right) \Leftrightarrow \Upsilon_{n}(z) \rightarrow e^{i n \alpha} \Upsilon_{n}(z)
$$

which can be regarded as chiral rotations of the fermionic coordinates of the $\mathcal{N}=2$ superspace (a diagonal group of automorphism group $S U(2)_{R}$ ). This $U(1)$ action is precisely the one in Refs. [26, 27] acting on fiber.

In Ref. [43, it was claimed that there exists a minimal extension of every four dimensional $\mathcal{N}=1$ supersymmetric nonlinear sigma model described by (2.16) to four dimensional $\mathcal{N}=2$ supersymmetric nonlinear sigma model described by (2.11). Indeed, it is easy to see that the action (2.11) involves $\mathcal{N}=1$ Kähler potential and can be regarded as an $\mathcal{N}=2$ extension. Representing $\Upsilon(\zeta)$ in the form $\Upsilon=\Phi+\zeta \Sigma+\mathcal{A}(\zeta)$ where $\mathcal{A}(\zeta)$ contains all the auxiliary superfields, the action (2.11) can be rewritten as

$$
S=\int d^{8} z\left\{\frac{1}{2 \pi i} \oint \frac{d \zeta}{\zeta} \exp \left(\mathcal{A} \frac{\partial}{\partial \Phi}+\overline{\mathcal{A}} \frac{\partial}{\partial \bar{\Phi}}\right) \exp \left[\zeta \Sigma \frac{\partial}{\partial \Phi}-\frac{1}{\zeta} \bar{\Sigma} \frac{\partial}{\partial \bar{\Phi}}\right] K(\Phi, \bar{\Phi})\right\}
$$

One can see that an extension of $\mathcal{N}=1$ model into $\mathcal{N}=2$ nonlinear sigma model can be obtained via (2.21) with corresponding $\mathcal{N}=1 \mathrm{Kähler}$ potential. However, since this action still includes the infinite tower of auxiliary superfields $\mathcal{A}$, we have to eliminate them by their equations of motion, in order to obtain the action in terms of physical superfields $\Phi$ and $\Sigma$ only. Their equations of motion read

$$
\frac{1}{2 \pi i} \oint \frac{d \zeta}{\zeta} \zeta^{n} \frac{\partial}{\partial \Upsilon_{*}^{i}} K\left(\Upsilon_{*}, \bar{\Upsilon}_{*}\right)=0, \quad n \geq 2
$$

with $\Upsilon_{*}(\zeta)$ denoting a solution.

In general, it is difficult to solve (2.22) exactly and the auxiliary fields can be eliminated at most perturbatively [43. However, it was claimed in Ref. [43] that one can exactly solve Eq. (2.22) if the following conditions are satisfied:

- The Kähler manifold is a homogeneous space, a coset space $G / H$, with an isometry $G$.

- The Kähler potential is invariant under the $\mathcal{N}=1 U(1)_{R}$ symmetry, defined by $\Phi \rightarrow e^{i \alpha} \Phi$ and $\Sigma \rightarrow e^{i \alpha} \Sigma$. 
The authors in Ref. 43 showed how to solve the equations (2.22) for the $\mathbf{C} P^{1}$ base manifold explicitly and constructed $\mathcal{N}=2$ supersymmetric nonlinear sigma models on the tangent $T \mathbf{C} P^{1}$ and the cotangent $T^{*} \mathbf{C} P^{1}$ bundles over $\mathbf{C} P^{1}$. They also wrote down the $T \mathbf{C} P^{n-1}$ model in Ref. 44. In the following, we give a comprehensive review of how to obtain the nonlinear sigma model with $T^{*} \mathbf{C} P^{n-1}$. Then, we consider the action with the tangent $T Q^{n-2}$ and cotangent $T^{*} Q^{n-2}$ bundles over quadric surface $Q^{n-2}=S O(n) /[S O(n-2) \times U(1)]$.

\section{3 (Co)tangent bundle over $\mathrm{C} P^{n-1}$}

The Kähler potential of $\mathbf{C} P^{n-1}$ nonlinear sigma model is given by ${ }^{7}$

$$
K\left(\Phi^{i}, \bar{\Phi}^{\bar{j}}\right)=r^{2} \ln \left(1+\frac{\left|\Phi^{i}\right|^{2}}{r^{2}}\right)
$$

where $\Phi^{i}(i=1, \cdots, n-1)$ are chiral superfields and $r$ is a real constant with mass dimension one. The potential for its $\mathcal{N}=2$ extension can be obtained by replacing $\Phi^{i}$ in (3.1) by the superfield $\Upsilon^{i}$. The equations of motion to the infinite auxiliary fields read from (2.22):

$$
\frac{1}{2 \pi i} \oint \frac{d \zeta}{\zeta} \zeta^{m} \frac{r^{2} \bar{\Upsilon}_{*}^{\bar{i}}}{r^{2}+\left|\Upsilon_{*}^{j}\right|^{2}}=0, \quad m \geq 2
$$

It is difficult to find a solution of Eq. (3.2) at arbitrary point of the base manifold $\mathbf{C} P^{n-1}$. However, one can readily find the solution at the origin $\Phi=0$ as

$$
\Upsilon_{0}^{i}=\Sigma_{0}^{i} \zeta, \quad \bar{\Upsilon}_{0}^{\bar{i}}=-\frac{\bar{\Sigma}_{0}^{\bar{i}}}{\zeta}
$$

Here $\Sigma_{0}^{i}$ is a tangent vector at the origin of $\Phi$. We need a solution $\Upsilon_{*}^{i}$ at $\Phi \neq 0$. In order to obtain it, one should take into account that Eq. (3.2) is invariant under the $S U(n)$ transformation because $\mathbf{C} P^{n-1}$ is a homogeneous space with $S U(n)$ isometry and the Kähler potential (3.1) is invariant under $S U(n)$ transformation up to a Kähler transformation. Then, applying $S U(n)$ transformation to the curve $\Upsilon_{0}^{i}(\zeta)$, one can obtain the solution $\Upsilon_{*}^{i}(\zeta)$ at $\Phi \neq 0$ (see Fig. 11).

Let $\phi^{i}=\left(x, y^{j}\right)^{T}(j=1, \cdots, n-1)$ be homogeneous coordinates of $\mathbf{C} P^{n-1}$. Infinistimal $S U(n)$ transformations for $\phi^{i}$ can be decomposed into [48]

$$
\begin{aligned}
\delta \phi^{i} & =\left(i \theta T+i \theta^{A} T_{A}+\bar{\epsilon}^{\bar{j}} E^{j}+\epsilon^{j} \bar{E}^{\bar{j}}\right) \phi^{i} \\
& =\left(\begin{array}{cc}
i \sqrt{\frac{2(n-1)}{n}} \theta & \bar{\epsilon}^{\bar{j}} \\
-\epsilon^{i} & -i \theta^{A} \rho\left(T_{A}\right)^{i j}-i \sqrt{\frac{2}{n(n-1)}} \theta \delta^{i j}
\end{array}\right)\left(\begin{array}{c}
x \\
y^{j}
\end{array}\right),
\end{aligned}
$$

\footnotetext{
${ }^{7}$ We take the notation that repeated indices mean the summation with respect to them even if they are at the same upper(down) positions. For instance, $A^{i j} B^{j} \equiv \sum_{j} A^{i j} B^{j}$. Throughout this paper, we respect upper and down indices as vector and covector, respectively.
} 


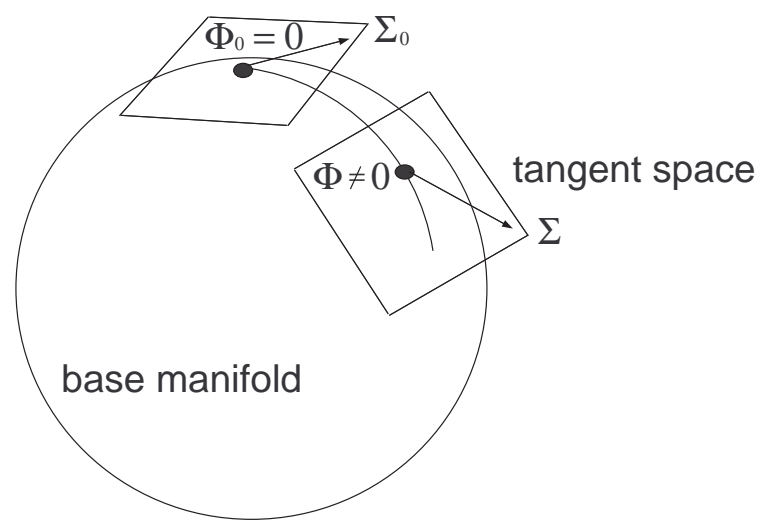

Figure 1: Base manifold $\mathbf{C} P^{n-1}$ with its tangent spaces at $\Phi=0$ and $\Phi \neq 0$. One can arrive at any point $\Phi \neq 0$ from $\Phi_{0}=0$ by $S U(n)$ transformation with parameters $\epsilon$ and $\bar{\epsilon}$ on the manifold. By using the transformation law for $\Phi$, the tangent vector at $\Phi \neq 0$ is also obtained.

where $T$ is the $U(1)$ generator, $E^{i}\left(\bar{E}^{\bar{i}}=\left(E^{i}\right)^{\dagger}\right)$ is $n-1$ raising (lowering) operators represented by upper (lower) triangle matrices, and $\rho\left(T_{A}\right)$ is an $n-1$ by $n-1$ matrix for the fundamental representation of $S U(n-1)$. From Eq. (3.4), one can obtain the action of the finite transformation on the homogeneous coordinate

$$
\phi^{i}=\left(\begin{array}{c}
x \\
y^{j}
\end{array}\right) \rightarrow\left(\begin{array}{c}
x \cos A+\frac{\bar{\epsilon} \cdot y \sin A}{A} \\
-\frac{\epsilon^{j} x \sin A}{A}+y^{j}-\frac{\epsilon^{j} \bar{\epsilon} \cdot y}{A^{2}}(1-\cos A)
\end{array}\right),
$$

with $A \equiv \epsilon^{i} \bar{\epsilon}_{\bar{i}}=\epsilon \cdot \bar{\epsilon}$. Here we take $\theta=\theta^{A}=0$ since such a parameter choice is sufficient to consider the point $\Phi \neq 0$. Using Eq. (3.5), one obtains the transformation law for inhomogeneous coordinates $\Phi^{i} \equiv r y^{i} / x(i=1, \cdots, n-1)$ of the $\mathbf{C} P^{n-1}$ as

$$
\Phi^{i}=f^{i}\left(\Phi_{0}^{j}\right)=\frac{\frac{-r \epsilon^{i} \sin A}{A \cos A}+\frac{\Phi_{0}^{i}}{\cos A}-\frac{\epsilon^{i}\left(\bar{\epsilon} \cdot \Phi_{0}\right)}{A^{2} \cos A}(1-\cos A)}{1+\frac{\bar{\epsilon} \cdot \Phi_{0} \sin A}{r A \cos A}} .
$$

Eq. (3.6) tells us a transformation law for the tangent vector $\Sigma^{i}$

$$
\Sigma^{i}=\left.\frac{\partial f^{i}(\Phi)}{\partial \Phi^{j}}\right|_{\Phi=\Phi_{0}} \Sigma_{0}^{j} .
$$

Substituting $\Phi_{0}=0$ into (3.6) and (3.7), one obtains the transformation law from the point $\Phi_{0}=0$ to $\Phi \neq 0$ as

$$
\begin{aligned}
\Phi^{i} & =f^{i}\left(\Phi_{0}=0\right)=-\frac{r \epsilon^{i} \sin A}{A \cos A}, \\
\Sigma^{i} & =\left.\frac{\partial f^{i}(\Phi)}{\partial \Phi^{j}}\right|_{\Phi=0} \Sigma_{0}^{j}=\left\{\frac{\delta^{i j}}{\cos A}-\frac{\epsilon^{i} \bar{\epsilon}}{A^{2} \cos ^{2} A}(\cos A-1)\right\} \Sigma_{0}^{j} \equiv V_{j}^{i}(\epsilon, \bar{\epsilon}) \Sigma_{0}^{j} .
\end{aligned}
$$


Here $V_{j}^{i}$ can be written in terms of $\Phi^{i}$ with (3.8) as

$$
V_{j}^{i}=\sqrt{1+\frac{\left|\Phi^{k}\right|^{2}}{r^{2}}}\left(\delta^{i j}-\frac{\Phi^{i} \bar{\Phi}^{\bar{j}}}{\left|\Phi^{l}\right|^{2}}\left(1-\sqrt{1+\frac{\left|\Phi^{m}\right|^{2}}{r^{2}}}\right)\right),
$$

and its inverse is given by

$$
\left(V^{-1}\right)^{i}{ }_{j}=\frac{1}{\sqrt{\frac{1+\left|\Phi^{k}\right|^{2}}{r^{2}}}}\left\{\delta^{i j}-\frac{\Phi^{i} \bar{\Phi}^{\bar{j}}}{\left|\Phi^{l}\right|^{2}}\left(1-\frac{1}{\sqrt{\frac{1+\left|\Phi^{m}\right|^{2}}{r^{2}}}}\right)\right\} .
$$

Taking that $\Upsilon^{i}$ follows the same transformation law with (3.6) into account, one finds

$$
\Upsilon_{*}^{i}=f^{i}\left(\Upsilon_{0}\right)=\frac{\frac{-r \epsilon^{i} \sin A}{A \cos A}+\frac{\Upsilon_{0}^{i}}{\cos A}-\frac{\epsilon^{i}\left(\bar{\epsilon} \cdot \Upsilon_{0}\right)}{A^{2} \cos A}(1-\cos A)}{1+\frac{\bar{\epsilon} \cdot \Upsilon_{0} \sin A}{r A \cos A}} .
$$

By using (3.3) and (3.8), Eq. (3.12) can be rewritten in terms of $\Phi^{i}$ and $\Sigma_{0}^{i}$ as

$$
\Upsilon_{*}^{i}=\frac{\Phi^{i}+D_{j}^{i} \Sigma_{0}^{j} \zeta}{1-\left(\bar{\Phi} \cdot \Sigma_{0}\right) \zeta / r^{2}}
$$

with

$$
D_{j}^{i} \equiv \sqrt{1+\frac{\left|\Phi^{k}\right|^{2}}{r^{2}}}\left(\delta^{i j}-\frac{\Phi^{i} \bar{\Phi}^{\bar{j}}}{\left|\Phi^{l}\right|^{2}}\right)+\frac{\Phi^{i} \bar{\Phi}^{\bar{j}}}{\left|\Phi^{k}\right|^{2}} .
$$

Using (3.9) and (3.11), we obtain a simple form of the solution

$$
\Upsilon_{*}^{i}=\frac{\Phi^{i}\left(1+\left|\Phi^{j}\right|^{2} / r^{2}\right)+\zeta\left\{\Sigma^{i}\left(1+\left|\Phi^{j}\right|^{2} / r^{2}\right)-\Phi^{i}(\bar{\Phi} \cdot \Sigma) / r^{2}\right\}}{1+\left|\Phi^{k}\right|^{2} / r^{2}-\zeta(\bar{\Phi} \cdot \Sigma) / r^{2}} .
$$

It can be easily checked that this is actually the solution of (3.2) by substituting (3.15) into (3.2).

Replacing $\Phi^{i}$ in the Kähler potential (3.1) by $\Upsilon_{*}^{i}$ in Eq. (3.15), we obtain

$$
K=\Psi+\bar{\Psi}+r^{2} \ln \left(1+\frac{\left|\Phi^{i}\right|^{2}}{r^{2}}\right)+r^{2} \ln \left\{1-\frac{1}{r^{2}\left(1+\frac{\left|\Phi^{j}\right|^{2}}{r^{2}}\right)}\left(\left|\Sigma^{i}\right|^{2}+\frac{|\Sigma \cdot \bar{\Phi}|^{2}}{r^{2}\left(1+\frac{\left|\Phi^{i}\right|^{2}}{r^{2}}\right)}\right)\right\}
$$

with $\Psi$ defined by

$$
\Psi \equiv-r^{2} \ln \left(1-\frac{\zeta}{r^{2}} \frac{\bar{\Phi} \cdot \Sigma}{1+\left|\Phi^{i}\right|^{2} / r^{2}}\right)
$$

After integrating over the projective coordinate and introducing the Kähler metric of $\mathbf{C} P^{n-1}$

$$
g_{i \bar{j}}=\frac{r^{2} \delta_{i \bar{j}}}{r^{2}+\left|\Phi^{k}\right|^{2}}-\frac{r^{2} \bar{\Phi}^{\bar{i}} \Phi^{j}}{\left(r^{2}+\left|\Phi^{k}\right|^{2}\right)^{2}},
$$


we finally obtain the following action

$$
S=\int d^{8} z\left\{r^{2} \ln \left(1+\frac{\left|\Phi^{i}\right|^{2}}{r^{2}}\right)+r^{2} \ln \left(1-\frac{1}{r^{2}} g_{i \bar{j}} \Sigma^{i} \bar{\Sigma}^{\bar{j}}\right)\right\} .
$$

This is the action of the nonlinear sigma model with the tangent bundle $T \mathbf{C} P^{n-1}$. Since the second term vanishes when $\Sigma=0$ holds, the first term is the Kähler potential of the base $\mathbf{C} P^{n-1}$ and the second term represents the tangent space. This form was obtained for $T \mathbf{C} P^{1}$ in Ref. [43] and for $T \mathbf{C} P^{n-1}$ in Ref. [44].

In order to obtain the cotangent bundle, we need dualize the complex linear superfields $\Sigma^{i}$ in the action (3.19) into chiral superfields $\psi_{i}$ being components of a cotangent vector via the Legendre transformation. The resultant action can be identified with a hyper-Kähler potential. The action can be dualized if we replace the action (3.19) by the following one:

$$
S=\int d^{8} z\left\{r^{2} \ln \left(1+\frac{\left|\Phi^{i}\right|^{2}}{r^{2}}\right)+r^{2} \ln \left(1-\frac{1}{r^{2}} g_{i \bar{j}} U^{i} \bar{U}^{\bar{j}}\right)+U^{i} \psi_{i}+\bar{U}^{\bar{i}} \bar{\psi}_{i}\right\},
$$

where $U^{i}$ is the complex unconstrained auxiliary superfields. By the construction, $U^{i}$ is a tangent vector at point $\Phi$ of the manifold and therefore $\psi_{i}$ is a one-form at the same point. Eliminating the auxiliary variables $U^{i}$ and $\bar{U}^{\bar{j}}$, with the aid of the equations of motion

$$
\begin{aligned}
0 & =\frac{\partial S}{\partial U^{i}}=\frac{-g_{i \bar{j}} \bar{U}^{\bar{j}}}{1-g_{i \bar{j}} U^{i} \bar{U}^{j} / r^{2}}+\psi_{i} \\
0 & =\frac{\partial S}{\partial \bar{U}^{i}}=\frac{-g_{j i} U^{j}}{1-g_{i \bar{j}} U^{i} \bar{U}^{j} / r^{2}}+\bar{\psi}_{\bar{i}}
\end{aligned}
$$

results in a purely chiral sigma model which is dually equivalent to the $\mathcal{N}=2$ supersymmetric model (3.19) and is defined on the cotangent bundle $T^{*} \mathbf{C} P^{n-1}$. The final result is [47, 52.

$$
S=\int d^{8} z\left\{r^{2} \ln \left(1+\frac{\left|\Phi^{i}\right|^{2}}{r^{2}}\right)-r^{2} \ln (f(\kappa))+2 r^{2} \frac{\kappa}{f(\kappa)}\right\}, \quad f(\kappa)=\frac{1}{2}(1+\sqrt{1+4 \kappa}),
$$

where $\kappa \equiv g^{i \bar{j}} \psi_{i} \bar{\psi}_{\bar{j}} / r^{2}$ and $g^{i \bar{j}}$ the inverse metric of $g_{i \bar{j}}$.

Here we solved the equation of motion (3.2) to remove the auxiliary fields. There is an another method to eliminate the infinite auxiliary fields, which is briefly reviewed in Appendix A.

\section{Construction of tangent bundle $T Q^{n-2}$}

In this section we construct the nonlinear sigma model with tangent bundle $T Q^{n-2} \simeq T\left[\frac{S O(n)}{S O(n-2) \times U(1)}\right]$. Here we use a strategy different from the last section, because it is difficult to solve the equations of motion (3.2) for elimination of auxiliary fields in this case. First we consider the $n=4$ case of $Q^{2}$ (called the Klein quadric) by noting the isomorphism $Q^{2} \simeq S O(4) /[S O(2) \times U(1)] \simeq$ $[S U(2) \times S U(2)] /[U(1) \times U(1)] \simeq \mathbf{C} P^{1} \times \mathbf{C} P^{1}$. We then solve the equations of motion for auxiliary fields in this $T Q^{2}$ case. We can show that the solution can be extended to the $T Q^{n-2}$ case, and then construct the $T Q^{n-2}$ model. 


\subsection{First look: $T Q^{2}$}

Let us start in the base manifold. Considering the isomorphism mentioned above, the Kähler potential of the nonlinear sigma model with $Q^{2}$ can be described by

$$
\begin{aligned}
K\left(\Phi^{i}, \bar{\Phi}^{j}\right) & =r^{2} \ln \left(1+\frac{\left|\Phi^{1}\right|^{2}}{r^{2}}\right)+r^{2} \ln \left(1+\frac{\left|\Phi^{2}\right|^{2}}{r^{2}}\right) \\
& =r^{2} \ln \left(1+\frac{\left|\Phi^{i}\right|^{2}}{r^{2}}+\frac{\left|\Phi^{1}\right|^{2}\left|\Phi^{2}\right|^{2}}{r^{4}}\right), \quad i=1,2 .
\end{aligned}
$$

Using the unitary transformation

$$
\left(\begin{array}{c}
\Phi^{1} \\
\Phi^{2}
\end{array}\right) \rightarrow \frac{1}{\sqrt{2}}\left(\begin{array}{cc}
1 & -i \\
1 & i
\end{array}\right)\left(\begin{array}{l}
\Phi^{1} \\
\Phi^{2}
\end{array}\right)
$$

we arrive at the Kähler potential (1.8) with $i=1,2$ for $Q^{2}$. The Kähler metric is then given by Eq. (1.9) with $i=1,2$.

Next we consider its tangent bundle. Replacing the chiral superfield $\Phi^{i}$ in Eq. (4.1) by the scalar multiplet $\Upsilon^{i}$, we arrive at the action of the tangent bundle:

$$
S=\int d^{8} z \frac{1}{2 \pi i} \oint \frac{d \zeta}{\zeta}\left\{K\left(\Upsilon^{1}, \bar{\Upsilon}^{1}\right)+K\left(\Upsilon^{2}, \bar{\Upsilon}^{2}\right)\right\}
$$

with

$$
K\left(\Upsilon^{i}, \bar{\Upsilon}^{i}\right)=r^{2} \ln \left(1+\frac{\left|\Upsilon^{i}\right|^{2}}{r^{2}}\right), \quad(\text { no sum })
$$

Since the calculation for the $\mathbf{C} P^{1}$ case in the previous section can be done independently for each term in (4.3), the resultant action is just a sum of the action (3.19) with a different kind of chiral and complex linear superfields:

$$
\begin{aligned}
S= & \int d^{8} z\left\{r^{2} \ln \left(1+\frac{\left|\Phi^{i}\right|^{2}}{r^{2}}+\frac{\left|\Phi^{1}\right|^{2}\left|\Phi^{2}\right|^{2}}{r^{4}}\right)\right. \\
& \left.+r^{2} \ln \left(1-\sum_{i=1}^{2} G_{i} \frac{\left|\Sigma^{i}\right|^{2}}{r^{2}}+\frac{1}{r^{4}} G_{1} G_{2}\left|\Sigma^{1}\right|^{2}\left|\Sigma^{2}\right|^{2}\right)\right\},
\end{aligned}
$$

where $G_{i}$ is the metric of $\mathbf{C} P^{1}$ given by

$$
G_{i}=\frac{1}{\left(1+\frac{\left|\Phi^{i}\right|^{2}}{r^{2}}\right)^{2}}, \quad(\text { no sum }) .
$$

In Eq. (4.5), the first term is the Kähler potential of the base manifold $Q^{2}$ and the second one is of tangent one. This form of the action is in particular coordinates and so it is better to rewrite it by geometrical quantities such as Kähler potential, metric and Riemann tensor. 
Indeed, as shown in (1.8), the first term is written by Kähler potential after performing unitarity transformation. Let us focus on the tangent vector sector (second logarithm). Taking that the tangent vector $\Sigma^{i}$ follows the same unitary transformation law with one of $\Phi^{i}$ into account, we find that the term $\sum_{i=1}^{2} G_{i}\left|\Sigma^{i}\right|^{2} / r^{2}$ is the form of $g_{i \bar{j}} \Sigma^{i} \bar{\Sigma}^{\bar{j}}$, where $g_{i \bar{j}}$ is a metric of quadric surface given in Eq. (1.9). The last term in the second logarithm, $G_{1} G_{2}\left|\Sigma^{1}\right|^{2}\left|\Sigma^{2}\right|^{2}$, can be rewritten by the covariant quantity by noting that it is in forth order in $\Sigma$ or $\bar{\Sigma}$. First, we calculate $\left(g_{i j} \Sigma^{i} \bar{\Sigma}^{\bar{j}}\right)^{2}$ in the frame taken in (4.5).

$$
\left(g_{i \bar{j}} \Sigma^{i} \bar{\Sigma}^{\bar{j}}\right)^{2}=G_{1}^{2}\left|\Sigma^{1}\right|^{4}+G_{2}^{2}\left|\Sigma^{2}\right|^{4}+2 G_{1} G_{2}\left|\Sigma^{1}\right|^{2}\left|\Sigma^{2}\right|^{2} .
$$

Another forth order term is

$$
\begin{aligned}
R_{i \bar{j} k \bar{l}} \Sigma^{i} \bar{\Sigma}^{\bar{j}} \Sigma^{k} \bar{\Sigma}^{\bar{l}} & =-\frac{2\left|\Sigma^{1}\right|^{4}}{r^{2}\left(1+\frac{\left|\Phi^{1}\right|^{2}}{r^{2}}\right)^{4}}-\frac{2\left|\Sigma^{2}\right|^{4}}{r^{2}\left(1+\frac{\left|\Phi^{2}\right|^{2}}{r^{2}}\right)^{4}} \\
& =-\frac{2}{r^{2}}\left(G_{1}^{2}\left|\Sigma^{1}\right|^{4}+G_{2}^{2}\left|\Sigma^{2}\right|^{4}\right)
\end{aligned}
$$

where $R_{i \bar{j} k \bar{l}}$ is Riemann tensor defined by $R_{i \bar{j} k \bar{l}}=\partial_{k} \partial_{\bar{l}} g_{i \bar{j}}-g^{m \bar{n}} \partial_{m} g_{i \bar{j}} \partial_{\bar{n}} g_{k \bar{l}}$. Using (4.7) and (4.8), we get the relation

$$
G_{1} G_{2}\left|\Sigma^{1}\right|^{2}\left|\Sigma^{2}\right|^{2}=\frac{1}{2}\left\{\left(g_{i \bar{j}} \Sigma^{i} \bar{\Sigma}^{\bar{j}}\right)^{2}+\frac{r^{2}}{2} R_{i \bar{j} k \bar{l}} \Sigma^{i} \bar{\Sigma}^{\bar{j}} \Sigma^{k} \bar{\Sigma}^{\bar{l}}\right\} .
$$

Putting all together, and performing the unitary transformation, we finally obtain the action of the tangent bundle $T Q^{2}$ over quadric surface $Q^{2}$

$$
\begin{aligned}
S= & \int d^{8} z\left[r^{2} \ln \left(1+\frac{\left|\Phi^{i}\right|^{2}}{r^{2}}+\frac{\left(\Phi^{i}\right)^{2}\left(\bar{\Phi}^{\bar{j}}\right)^{2}}{4 r^{4}}\right)\right. \\
& \left.+r^{2} \ln \left\{1-\frac{1}{r^{2}} g_{i \bar{j}} \Sigma^{i} \bar{\Sigma}^{\bar{j}}+\frac{1}{2 r^{4}}\left(\left(g_{i \bar{j}} \Sigma^{i} \bar{\Sigma}^{\bar{j}}\right)^{2}+\frac{r^{2}}{2} R_{i \bar{j} k \bar{l}} \Sigma^{i} \bar{\Sigma}^{\bar{j}} \Sigma^{k} \bar{\Sigma}^{\bar{l}}\right)\right\}\right] .
\end{aligned}
$$

There appear the fourth order terms of the tangent vector. This is a particular form of the general expression of the hyper-Kähler sigma models suggested in (2.47) in Ref. [43. The method which we use is simpler and easier than solving the equation of motion (3.2) as performed in the previous section, in which one may suffer from rewriting the transformation parameters $\epsilon^{i}$ and $\bar{\epsilon}^{i}$ in terms of $\Phi^{i}$ as has been done in (3.15) and also from finding the form of the tangent bundle action (4.10). In the following subsection, we solve the equations of motion for auxiliary fields for $T Q^{n-2}$ case as the same with the $T \mathbf{C} P^{n-1}$ case. Then, we show that the tangent bundle action (4.10) is also valid in $T Q^{n-2}$ with simply extending the index $i$ to run from 1 to $n-2$.

\subsection{Solving the equations of motion and deriving $T Q^{n-2}$ action}

We start with $\mathcal{N}=2$ extension of the Kähler potential of $Q^{n-2}$

$$
K\left(\Upsilon^{i}, \bar{\Upsilon}^{\bar{j}}\right)=r^{2} \ln \left(1+\frac{\left|\Upsilon^{i}\right|^{2}}{r^{2}}+\frac{\left(\Upsilon^{i}\right)^{2}\left(\bar{\Upsilon}^{\bar{j}}\right)^{2}}{4 r^{4}}\right)
$$


The equation of motion for auxiliary fields of the polar multiplet reads

$$
\frac{1}{2 \pi i} \oint_{C} \frac{d \zeta}{\zeta} \zeta^{m} \frac{r^{2} \bar{\Upsilon}_{*}^{i}+\frac{1}{2} \Upsilon_{*}^{i}\left(\bar{\Upsilon}_{*}^{\bar{j}}\right)^{2}}{r^{4}+r^{2}\left|\Upsilon_{*}^{k}\right|^{2}+\frac{1}{4}\left(\Upsilon_{*}^{k}\right)^{2}\left(\bar{\Upsilon}_{*}^{\bar{l}}\right)^{2}}=0, \quad m \geq 2
$$

It is easy to check that the same solution with (3.3) also satisfies this equation. We can obtain the solution at $\Phi \neq 0$ by applying the finite $S O(n)$ transformation to the curve (3.3) since (4.12) is $S O(n)$ invariant.

In the following we again focus on the $n=4$ case, $T Q^{2}$, for a while. Using Eq. (1.4) leads to the finite $S O(4)$ transformation law for the homogeneous coordinates as

$$
\begin{aligned}
x= & \frac{1}{2}\left(\cos \beta_{+}+\cos \beta_{-}\right) x_{0}+\frac{\beta_{+}\left(\bar{\gamma}_{+} \cdot y_{0}\right) \sin \beta_{-}+\beta_{-}\left(\bar{\gamma}_{-} \cdot y_{0}\right) \sin \beta_{+}}{2 \lambda \beta_{+} \beta_{-}} \\
& -\frac{\sqrt{\left(\bar{\epsilon}^{\bar{i}}\right)^{2}}\left(\cos \beta_{+}-\cos \beta_{-}\right)}{2 \sqrt{\left(\epsilon^{i}\right)^{2}}} z_{0}, \\
y^{i}= & \frac{1}{2 \lambda}\left[\left(\cos \beta_{-}-\cos \beta_{+}\right)\left\{\epsilon^{i}\left(\bar{\epsilon} \cdot y_{0}\right)-y_{0}^{i}(\epsilon \cdot \bar{\epsilon})+\bar{\epsilon}\left(\epsilon \cdot y_{0}\right)\right\}+\lambda\left(\cos \beta_{+}+\cos \beta_{-}\right) y_{0}^{i}\right] \\
& -\frac{\beta_{+} \gamma_{+}^{i} \sin \beta_{-}+\beta_{-} \gamma_{-}^{i} \sin \beta_{+}}{2 \lambda \beta_{+} \beta_{-}} x_{0}-\frac{\beta_{+} \bar{\gamma}_{+}^{\bar{i}} \sin \beta_{-}+\beta_{-} \bar{\gamma}_{-}^{i} \sin \beta_{+}}{2 \lambda \beta_{+} \beta_{-}} z_{0},
\end{aligned}
$$

where

$$
\begin{aligned}
\lambda & \equiv \sqrt{\left(\epsilon^{i}\right)^{2}} \sqrt{\left(\bar{\epsilon}^{j}\right)^{2}}, \\
\beta_{+} & \equiv \sqrt{\epsilon \cdot \bar{\epsilon}-\lambda}, \quad \beta_{-} \equiv \sqrt{\epsilon \cdot \bar{\epsilon}+\lambda} \\
\gamma_{+}^{i} & \equiv\left(\epsilon^{j}\right)^{2} \bar{\epsilon}^{\bar{i}}+\epsilon^{i} \lambda, \quad \gamma_{-}^{i} \equiv-\left(\epsilon^{j}\right)^{2} \bar{\epsilon}^{\bar{i}}+\epsilon^{i} \lambda .
\end{aligned}
$$

Using the definition of the inhomogeneous coordinates of quadric surface $\Phi^{i} \equiv r y^{i} / x$, the transformation laws for $\Phi^{i}$ and $\Sigma^{i}$ are given by

$$
\begin{aligned}
\Phi^{i} & =\left.r \frac{y^{i}}{x}\right|_{\Phi_{0}=0}=-r \frac{\beta_{+} \gamma_{+}^{i} \sin \beta_{-}+\beta_{-} \gamma_{-}^{i} \sin \beta_{+}}{\lambda \beta_{+} \beta_{-}\left(\cos \beta_{+}+\cos \beta_{-}\right)}, \\
\Sigma^{i} & =\left[\frac{\left(\cos \beta_{-}-\cos \beta_{+}\right)\left(\epsilon^{i} \bar{\epsilon}^{\bar{j}}-\delta^{i j}(\epsilon \cdot \bar{\epsilon})+\bar{\epsilon}^{\bar{i}} \epsilon^{j}\right)}{\lambda\left(\cos \beta_{+}+\cos \beta_{-}\right)}+\delta^{i j}+\frac{\Phi^{i} \bar{\Phi}^{\bar{j}}}{r^{2}}\right] \Sigma_{0}^{j} \\
& \equiv\left(V_{Q}\right)^{i}{ }_{j} \Sigma_{0}^{j} .
\end{aligned}
$$

Here, in the first line in Eq. (4.19), we have replaced the third term written by $\epsilon$ and $\bar{\epsilon}$ with $\Phi^{i}$ and $\bar{\Phi}^{j}$ defined in (4.18). Since the transformation law for $\Upsilon^{i}$ is the same with one of $\Phi^{i}$, we can obtain the $\Upsilon_{*}^{i}$ at non-zero value of $\Phi^{i}$ from (4.13) and (4.14) as

$$
\begin{aligned}
\Upsilon_{*}^{i}= & {\left[\frac{\cos \beta_{+}\left\{-\epsilon^{i}\left(\bar{\epsilon} \cdot \Upsilon_{0}\right)+\beta_{-}^{2} \Upsilon_{0}^{i}-\bar{\epsilon}^{\bar{i}}\left(\epsilon \cdot \Upsilon_{0}\right)\right\}+\cos \beta_{-}\left\{\epsilon^{i}\left(\bar{\epsilon} \cdot \Upsilon_{0}\right)-\beta_{+}^{2} \Upsilon_{0}^{i}+\bar{\epsilon}^{i}\left(\epsilon \cdot \Upsilon_{0}\right)\right\}}{\lambda\left(\cos \beta_{+}+\cos \beta_{-}\right)}\right.} \\
& \left.-r \frac{\beta_{+} \gamma_{+}^{i} \sin \beta_{-}+\beta_{-} \gamma_{-}^{i} \sin \beta_{+}}{\lambda \beta_{+} \beta_{-}\left(\cos \beta_{+}+\cos \beta_{-}\right)}+\frac{\beta_{+} \bar{\gamma}_{+}^{\bar{i}} \sin \beta_{-}+\beta_{-} \bar{\gamma}_{-}^{\bar{i}} \sin \beta_{+}}{2 r \lambda \beta_{+} \beta_{-}\left(\cos \beta_{+}+\cos \beta_{-}\right)}\left(\Upsilon_{0}^{l}\right)^{2}\right]
\end{aligned}
$$




$$
\times\left[1+\frac{\beta_{+}\left(\bar{\gamma}_{+} \cdot \Upsilon_{0}\right) \sin \beta_{-}+\beta_{-}\left(\bar{\gamma}_{-} \cdot \Upsilon_{0}\right) \sin \beta_{+}}{r \lambda \beta_{+} \beta_{-}\left(\cos \beta_{+}+\cos \beta_{-}\right)}+\frac{\sqrt{\left(\bar{\epsilon}^{\bar{i}}\right)^{2}}\left(\cos \beta_{+}-\cos \beta_{-}\right)}{2 r^{2} \sqrt{\left(\epsilon^{j}\right)^{2}}\left(\cos \beta_{+}+\cos \beta_{-}\right)}\left(\Upsilon_{0}^{k}\right)^{2}\right]^{-1}
$$

Let us rewrite Eq. (4.20) in terms of $\Phi^{i}$ and $\Sigma^{i}$. In order to do that, we need some formulas. Using the expression (4.18) we have

$$
\begin{aligned}
\left(\Phi^{i}\right)^{2} & =\frac{2 r^{2}\left(\epsilon^{i}\right)^{2}\left(\cos \beta_{+}-\cos \beta_{-}\right)}{\lambda\left(\cos \beta_{+}+\cos \beta_{-}\right)}, \\
\left(\bar{\Phi}^{\bar{i}}\right)^{2} & =\frac{2 r^{2}(\overline{\bar{\epsilon}})^{2}\left(\cos \beta_{+}-\cos \beta_{-}\right)}{\lambda\left(\cos \beta_{+}+\cos \beta_{-}\right)} .
\end{aligned}
$$

Multiplying (4.21) and (4.22), we get

$$
\left(\Phi^{i}\right)^{2}\left(\bar{\Phi}^{\bar{j}}\right)^{2}=\frac{4 r^{4}\left(\cos \beta_{+}-\cos \beta_{-}\right)^{2}}{\left(\cos \beta_{+}+\cos \beta_{-}\right)^{2}} .
$$

Dividing (4.21) by (4.22), we find

$$
\frac{\sqrt{\left(\bar{\epsilon}^{\bar{i}}\right)^{2}}}{\sqrt{\left(\epsilon^{j}\right)^{2}}}=\frac{\sqrt{\left(\bar{\Phi}^{\bar{i}}\right)^{2}}}{\sqrt{\left(\Phi^{j}\right)^{2}}}
$$

Using (4.18), (4.23) and (4.24), Eq. (4.20) can be rewritten as

$$
\begin{aligned}
\Upsilon *^{i}= & {\left[\frac{\cos \beta_{+}\left\{-\epsilon^{i}\left(\bar{\epsilon} \cdot \Upsilon_{0}\right)+\beta_{-}^{2} \Upsilon_{0}^{i}-\bar{\epsilon}^{\bar{i}}\left(\epsilon \cdot \Upsilon_{0}\right)\right\}+\cos \beta_{-}\left\{\epsilon^{i}\left(\bar{\epsilon} \cdot \Upsilon_{0}\right)-\beta_{+}^{2} \Upsilon_{0}^{i}+\bar{\epsilon}^{\bar{i}}\left(\epsilon \cdot \Upsilon_{0}\right)\right\}}{\lambda\left(\cos \beta_{+}+\cos \beta_{-}\right)}\right.} \\
& \left.+\Phi^{i}-\frac{1}{2 r^{2}} \bar{\Phi}^{\bar{i}}\left(\Upsilon_{0}^{j}\right)^{2}\right]\left[1-\frac{\bar{\Phi} \cdot \Upsilon_{0}}{r^{2}}+\frac{\left(\bar{\Phi}^{\bar{i}}\right)^{2}}{4 r^{4}}\left(\Upsilon_{0}^{k}\right)^{2}\right]^{-1}
\end{aligned}
$$

Further, using (4.19) with $\Upsilon_{0}^{i}=\zeta \Sigma_{0}^{i}$, we obtain a simple form

$$
\Upsilon *^{i}=\frac{\Phi^{i}+\zeta\left(\Sigma^{i}-\frac{\Phi^{i}\left(\bar{\Phi}\left(V_{Q}^{-1}\right) \Sigma\right)}{r^{2}}\right)-\zeta^{2} \frac{\bar{\Phi}^{\bar{i}}\left(\Sigma^{t}\left(V_{Q}^{-1}\right)^{t} V_{Q}^{-1} \Sigma\right)}{2 r^{2}}}{1-\zeta \frac{\left(\bar{\Phi} V_{Q}^{-1} \Sigma\right)}{r^{2}}+\zeta^{2} \frac{\left(\bar{\Phi}^{\bar{k}}\right)^{2}\left(\Sigma^{t}\left(V_{Q}^{-1}\right)^{t} V_{Q}^{-1} \Sigma\right)}{4 r^{4}}} .
$$

To complete the work, we have to find the expression of $V_{Q}^{i j}$ in terms of $\Phi^{i}$. The result is

$$
\begin{aligned}
V_{Q} & =\left(\begin{array}{cc}
1+\frac{\left|\Phi^{k}\right|^{2}}{2 r^{2}} & \frac{\Phi^{1} \bar{\Phi}^{2}-\bar{\Phi}^{\overline{1}} \Phi^{2}}{2 r^{2}} \\
-\frac{\Phi^{1} \bar{\Phi}^{2}-\bar{\Phi}^{1} \Phi^{2}}{2 r^{2}} & 1+\frac{\left|\Phi^{k}\right|^{2}}{2 r^{2}}
\end{array}\right), \\
V_{Q}^{-1} & =L^{-1}\left(\begin{array}{cc}
1+\frac{\left|\Phi^{k}\right|^{2}}{2 r^{2}} & -\frac{\Phi^{1} \bar{\Phi}^{\overline{2}}-\bar{\Phi}^{\overline{1}} \Phi^{2}}{2 r^{2}} \\
\frac{\Phi^{1} \bar{\Phi}^{2}-\bar{\Phi}^{1} \Phi^{2}}{2 r^{2}} & 1+\frac{\left|\Phi^{k}\right|^{2}}{2 r^{2}}
\end{array}\right),
\end{aligned}
$$


with

$$
L \equiv 1+\frac{\left|\Phi^{i}\right|^{2}}{r^{2}}+\frac{\left(\Phi^{i}\right)^{2}\left(\bar{\Phi}^{\bar{j}}\right)^{2}}{4 r^{4}}
$$

Eq. (4.28) leads $\left(V_{Q}^{-1 t} V_{Q}^{-1}\right)^{i j}=L^{-1} \delta^{i j}$ and we obtain the final form of the solution

$$
\Upsilon_{*}^{i}=\frac{\Phi^{i}+\zeta\left\{\Sigma^{i}-\frac{\Phi^{i}}{r^{2} L}\left(\bar{\Phi} \cdot \Sigma+\frac{(\Phi \cdot \Sigma)\left(\bar{\Phi}^{j}\right)^{2}}{2 r^{2}}\right)\right\}-\zeta^{2} \frac{\bar{\Phi}^{i}\left(\Sigma^{j}\right)^{2}}{2 r^{2} L}}{1-\frac{\zeta}{r^{2} L}\left\{\bar{\Phi} \cdot \Sigma+\frac{(\Phi \cdot \Sigma)\left(\bar{\Phi}^{k}\right)^{2}}{2 r^{2}}\right\}+\zeta^{2} \frac{\left(\bar{\Phi}^{k}\right)^{2}\left(\Sigma^{l}\right)^{2}}{4 r^{4} L}}
$$

This solution satisfies the equation of motion (4.12) for the $T Q^{2}$ case of $n=4$.

Let us turn back to general $n$. When one tries to derive the solution $\Upsilon_{*}^{i}$ for higher dimensional case $(n>4)$, it might be problematic to convert the transformation parameters $\epsilon^{i}$ and $\bar{\epsilon}^{\bar{i}}$ in $V_{Q}$ and $V_{Q}^{-1}$ into the base coordinates $\Phi$ and eventually to find the final form of the solution $\Upsilon_{*}$. But fortunately if one respects the solution (3.12) of $n=4$ by making the index $i$ run from 1 to $n-2$, it becomes a solution for general $n$. Indeed, one can straightforwardly check that it satisfies the equations of motion (4.12) by substituting Eq. (4.30) into (4.12):

$$
\begin{aligned}
& \frac{1}{2 \pi i} \oint_{C} \frac{d \zeta}{\zeta} \zeta^{m} \frac{r^{2} \bar{\Upsilon}_{*}^{i}+\frac{1}{2} \Upsilon_{*}^{i}\left(\bar{\Upsilon}_{*}^{j}\right)^{2}}{r^{4}+r^{2}\left|\Upsilon_{*}^{k}\right|^{2}+\frac{1}{4}\left(\Upsilon_{*}^{k}\right)^{2}\left(\bar{\Upsilon}_{*}^{\bar{l}}\right)^{2}} \\
& =\frac{1}{2 \pi i} \oint_{C} \frac{d \zeta}{\zeta} \zeta^{m-2} \frac{8 r^{4}}{g(\Phi, \Sigma)}\left[4 r^{4}\left(\bar{\Sigma}^{i}\right)^{2} Y^{i}-8 r^{2} \zeta(\bar{\Phi} \cdot \bar{\Sigma})\left(r^{2}+\left|\Phi^{j}\right|^{2}\right) Y^{i}+\zeta^{2}\left(\Phi^{j}\right)^{2}\left(\bar{\Phi}^{k}\right)^{4} Y^{i}\right. \\
& +8 r^{4} \zeta^{2}\left(r^{2}+\left|\Phi^{j}\right|^{2}-\zeta(\bar{\Phi} \cdot \Sigma)\right) \bar{Y}^{i}+2\left(\bar{\Phi}^{j}\right)^{2} r^{2} \zeta\left\{2(\Phi \cdot \bar{\Sigma}) Y^{i}+\zeta\left(2\left|\Phi^{j}\right|^{2} Y^{i}+2 r^{2} Y^{i}+\left(\Phi^{j}\right)^{2} \bar{Y}^{i}\right.\right. \\
& \\
& \left.\left.\left.-2 \zeta(\Phi \cdot \Sigma) \bar{Y}^{i}+\zeta^{2}\left(\Sigma^{j}\right)^{2} \bar{Y}^{i}\right)\right\}\right]
\end{aligned}
$$

where

$$
Y^{i}=L \Phi^{i}+\zeta\left\{L \Sigma^{i}-\frac{\Phi^{i}}{r^{2}}\left(\bar{\Phi} \cdot \Sigma+\frac{(\Phi \cdot \Sigma)\left(\bar{\Phi}^{j}\right)^{2}}{2 r^{2}}\right)\right\}-\zeta^{2} \frac{\bar{\Phi}^{i}\left(\Sigma^{j}\right)^{2}}{2 r^{2}}
$$

Here the denominator in the integrand can be written as $\zeta^{3} g(\Phi, \Sigma)$ and the function of $\Phi$ and $\Sigma, g(\Phi, \Sigma)$ can be factored out from the integral. Recalling that $m \geq 2$, the numerator is a polynomial starting from an order of $\zeta^{0}$ in the $m=2$ case. In this case, the terms greater than the first order of $\zeta$ vanish because of the residue theorem and the terms in the order of $\zeta^{0}$ only remain. However, they cancel and the integral becomes zero. In the case $m \geq 3$, the integral are trivially zero since all terms in the numerator starts from first order of $\zeta$.

Substituting the solution (4.30), with the index $i$ running from 1 to $n-2$, into the Kähler potential (4.11), we can derive the nonlinear sigma model action with $T Q^{n-2}$ space:

$$
\begin{aligned}
S= & \int d^{8} z \frac{1}{2 \pi i} \oint \frac{d \zeta}{\zeta}\left[\Psi+\bar{\Psi}+r^{2} \ln \left(1+\frac{\left|\Phi^{i}\right|^{2}}{r^{2}}+\frac{\left(\Phi^{i}\right)^{2}\left(\bar{\Phi}^{\bar{j}}\right)^{2}}{r^{4}}\right)\right. \\
& \left.+r^{2} \ln \left\{1-\frac{1}{r^{2}} g_{i \bar{j}} \Sigma^{i} \bar{\Sigma}^{\bar{j}}+\frac{1}{2 r^{2}}\left(\left(g_{i \bar{j}} \Sigma^{i} \bar{\Sigma}^{\bar{j}}\right)^{2}+\frac{r^{2}}{2} R_{i \bar{j} k \bar{l}} \Sigma^{i} \bar{\Sigma}^{\bar{j}} \Sigma^{k} \bar{\Sigma}^{\bar{l}}\right)\right\}\right]
\end{aligned}
$$


with $\Psi$ defined by

$$
\Psi=-r^{2} \ln \left\{1-\frac{\zeta}{r^{2} L}\left(\bar{\Phi} \cdot \Sigma+\frac{(\Phi \cdot \Sigma)\left(\bar{\Phi}^{i}\right)^{2}}{2 r^{2}}\right)+\zeta^{2} \frac{\left(\bar{\Phi}^{i}\right)^{2}\left(\Sigma^{j}\right)^{2}}{4 r^{4} L}\right\}
$$

After performing the $\zeta$-integration, we arrive at the same form with (4.10) with the index $i$ running from 1 to $n-2$.

In Appendix D, we show this action in $n=3$ coincides with the result in section 3 due to isomorphism $Q^{1} \simeq \mathbf{C} P^{1}$.

\section{Cotangent bundle $T^{*} Q^{n-2}$}

Here we consider the nonlinear sigma model action with the cotangent bundle over $Q^{n-2}, T^{*} Q^{n-2}$ (hyper-Kähler potential). To obtain the cotangent bundle, we need dualize the tangent vector $\Sigma^{i}$ into cotangent one $\psi_{i}$. In order to do that, let us consider the following Lagrangian instead of the tangent bundle action (4.10):

$$
\begin{aligned}
S= & \int d^{8} z\left[r^{2} \ln \left(1+\frac{\left|\Phi^{i}\right|^{2}}{r^{2}}+\frac{\left(\Phi^{i}\right)^{2}\left(\bar{\Phi}^{\bar{j}}\right)^{2}}{4 r^{4}}\right)\right. \\
& \left.+r^{2} \ln \left\{1-\frac{1}{r^{2}} g_{i \bar{j}} U^{i} \bar{U}^{\bar{j}}+\frac{1}{2 r^{4}}\left(\left(g_{i \bar{j}} U^{i} \bar{U}^{\bar{j}}\right)^{2}+\frac{r^{2}}{2} R_{i \bar{j} k \bar{l}} U^{i} \bar{U}^{\bar{j}} U^{k} \bar{U}^{\bar{l}}\right)\right\}+U^{i} \psi_{i}+\bar{U}^{i} \bar{\psi}_{\bar{i}}\right]
\end{aligned}
$$

where $U^{i}$ and $\bar{U}^{\bar{j}}$ are unconstrained superfields, regarded as a tangent vector, and $\psi_{i}$ and $\bar{\psi}_{\bar{j}}$ are chiral superfields, regarded as a cotangent vector. Equation of motion of $\psi$ brings back to original Lagrangian (4.10). On the other hand, varying with respect to $U^{i}$ and $\bar{U}^{\bar{j}}$, we obtain

$$
\begin{aligned}
\psi_{i} & =\frac{g_{i \bar{j}} \bar{U}^{\bar{j}}-\frac{1}{r^{2}} g_{i \bar{j}} \overline{U^{j}} \rho-\frac{1}{2} R_{i \bar{j} k \bar{l}} \bar{U}^{j} U^{k} \bar{U}^{\bar{l}}}{1-\frac{\rho}{r^{2}}+\frac{\rho^{2}}{2 r^{4}}+\frac{\sigma}{4 r^{2}}}, \\
\bar{\psi}_{\bar{j}} & =\frac{g_{i \bar{j}} U^{i}-\frac{1}{r^{2}} g_{i \bar{j}} U^{i} \rho-\frac{1}{2} R_{i \bar{j} k \bar{l}} U^{i} U^{k} \bar{U}^{\bar{l}}}{1-\frac{\rho}{r^{2}}+\frac{\rho^{2}}{2 r^{4}}+\frac{\sigma}{4 r^{2}}}
\end{aligned}
$$

where

$$
\rho \equiv g_{i \bar{j}} U^{i} \bar{U}^{\bar{j}}, \quad \sigma \equiv R_{i \bar{j} k \bar{l}} U^{i} \bar{U}^{\bar{j}} U^{k} \bar{U}^{\bar{l}}
$$

Multiplying (5.2) and (5.3) by $U^{i}$ and $\bar{U}^{\bar{i}}$, respectively, we get

$$
U^{i} \psi_{i}=\bar{U}^{\bar{i}} \bar{\psi}_{\bar{i}}=\frac{\rho-\frac{\rho^{2}}{r^{2}}-\frac{\sigma}{2}}{1-\frac{\rho}{r^{2}}+\frac{\rho^{2}}{2 r^{4}}+\frac{\sigma}{4 r^{2}}} .
$$


Substituting Eqs. (5.2), (5.3) and (5.5) into the action (5.1), one can see that the action (5.1) is a function of the covariant quantities $\rho$ and $\sigma$ along with base manifold coordinates $\Phi^{i}$. In the following, we show that $\rho$ and $\sigma$ are written by quantities

$$
\xi \equiv g^{i \bar{j}} \psi_{i} \bar{\psi}_{\bar{j}}, \quad \chi \equiv R^{\bar{i} j \bar{k} l} \bar{\psi}_{\bar{i}} \psi_{j} \bar{\psi}_{\bar{k}} \psi_{l}
$$

which are possible scalars in terms of cotangent vectors $\psi_{i}$ and $\bar{\psi}_{\bar{i}}$. In order to do that, first we substitute (5.2) and (5.3) into Eq. (5.6), and show that $\xi$ and $\chi$ are written in terms of $\rho$ and $\sigma$ with the help of the identities between metric and Riemann tensor. Then, solving them with respect to $\rho$ and $\sigma$, and substituting the solution into the action (5.1), we obtain the cotangent bundle action. In the following, we give an explicit calculation on the $T^{*} Q^{2}$ case. The detailed derivation of the identities in this case is given in Appendix B. The resultant equations after substituting (5.2) and (5.3) into (5.6) is

$$
\begin{aligned}
& \xi=g^{i \bar{j}} \psi_{i} \bar{\psi}_{\bar{j}}=\left(1-\frac{\rho}{r^{2}}+\frac{\rho^{2}}{2 r^{4}}+\frac{\sigma}{4 r^{2}}\right)^{-2}\left(\rho-\frac{2 \rho^{2}}{r^{2}}-\sigma+\frac{\rho^{3}}{2 r^{4}}+\frac{\rho \sigma}{4 r^{2}}\right), \\
& \chi=R^{\bar{i} j \bar{k} l} \bar{\psi}_{\bar{i}} \psi_{j} \bar{\psi}_{\bar{k}} \psi_{l}=\left(1-\frac{\rho}{r^{2}}+\frac{\rho^{2}}{2 r^{4}}+\frac{\sigma}{4 r^{2}}\right)^{-4} \\
& \times\left(\sigma+\frac{2 \rho \sigma}{r^{2}}-\frac{6 \rho^{2} \sigma}{r^{4}}+\frac{2 \rho^{3} \sigma}{r^{6}}+\frac{\rho^{4} \sigma}{4 r^{8}}+\frac{\sigma^{3}}{16 r^{4}}+\frac{\rho^{2} \sigma^{2}}{4 r^{6}}-\frac{6 \rho^{4}}{r^{6}}\right. \\
& \left.+\frac{2 \rho^{5}}{r^{8}}-\frac{3 \sigma^{2}}{2 r^{2}}+\frac{\rho \sigma^{2}}{2 r^{4}}+\frac{4 \rho^{3}}{r^{4}}\right) \text {. }
\end{aligned}
$$

Solving Eqs. (5.7) and (5.8) with respect to $\rho$ and $\sigma$ we obtain

$$
\begin{aligned}
\rho= & 2 r^{2}\left(1-\frac{1}{1+\sqrt{1+\frac{2 \xi}{r^{2}}+2 \sqrt{-\frac{\xi^{2}}{r^{4}}-\frac{\chi}{r^{2}}}}}-\frac{1}{1+\sqrt{1+\frac{2 \xi}{r^{2}}-2 \sqrt{-\frac{\xi^{2}}{r^{4}}-\frac{\chi}{r^{2}}}}}\right), \\
\sigma= & -4 r^{2}\left\{1+\frac{2}{\left(1+\sqrt{1+\frac{2 \xi}{r^{2}}-2 \sqrt{-\frac{\xi^{2}}{r^{4}}-\frac{\chi}{r^{2}}}}\right)^{2}}+\frac{2}{\left(1+\sqrt{1+\frac{2 \xi}{r^{2}}+2 \sqrt{-\frac{\xi^{2}}{r^{4}}-\frac{\chi}{r^{2}}}}\right)^{2}}\right. \\
& \left.-\frac{2}{1+\sqrt{1+\frac{2 \xi}{r^{2}}-2 \sqrt{-\frac{\xi^{2}}{r^{4}}-\frac{\chi}{r^{2}}}}}\right\} .
\end{aligned}
$$

Substituting back (5.9) and (5.10) into the action (5.1), we obtain the action for the contangent bundle $T^{*} Q^{2}$

$$
S=\int d^{8} z\left\{r^{2} \ln \left(1+\frac{\left|\Phi^{i}\right|^{2}}{r^{2}}+\frac{\left(\Phi^{i}\right)^{2}\left(\bar{\Phi}^{\bar{j}}\right)^{2}}{4 r^{2}}\right)\right.
$$




$$
\begin{aligned}
& -r^{2} \ln \left(\frac{1}{2}+\frac{1}{2} \sqrt{1+\frac{2 \xi}{r^{2}}+2 \sqrt{-\frac{\xi^{2}}{r^{4}}-\frac{\chi}{r^{2}}}}\right)-r^{2} \ln \left(\frac{1}{2}+\frac{1}{2} \sqrt{1+\frac{2 \xi}{r^{2}}-2 \sqrt{-\frac{\xi^{2}}{r^{4}}-\frac{\chi}{r^{2}}}}\right) \\
& \left.+\frac{\xi+r^{2} \sqrt{-\frac{\xi^{2}}{r^{4}}-\frac{\chi}{r^{2}}}}{\frac{1}{2}+\frac{1}{2} \sqrt{1+\frac{2 \xi}{r^{2}}+2 \sqrt{-\frac{\xi^{2}}{r^{4}}-\frac{\chi}{r^{2}}}}}+\frac{\xi-r^{2} \sqrt{-\frac{\xi^{2}}{r^{4}}-\frac{\chi}{r^{2}}}}{\frac{1}{2}+\frac{1}{2} \sqrt{1+\frac{2 \xi}{r^{2}}-2 \sqrt{-\frac{\xi^{2}}{r^{4}}-\frac{\chi}{r^{2}}}}}\right\}
\end{aligned}
$$

This action is of $T^{*} Q^{2}$. When one tries to derive the cotangent bundle action of $T^{*} Q^{n-2}$ case, one comes across difficulty in deriving the identities for cotangent bundle action for $T^{*} Q^{n-2}$, as shown in Appendix B. However, we believe that this form of the action would be also valid in $T Q^{n-2}$ case with extending the index to run from 1 to $n-2$, since the action is written in geometric quantities such as Kähler potential, metric and Riemann tensor as in the tangent bundle case.

One might consider a possibility that invariant quantities higher in $\psi$ such like $\left(g^{i \bar{j}} \psi_{i} \bar{\psi}_{\bar{j}}\right)^{3}$ and $\left(g^{i \bar{j}} \psi_{i} \bar{\psi}_{\bar{j}}\right)\left(R^{\bar{k} l \bar{m} n} \bar{\psi}_{\bar{k}} \psi_{l} \bar{\psi}_{\bar{m}} \psi_{n}\right)$ together with $\xi$ and $\chi$ appear in the cotangent bundle action. However, one can confirm by deriving the cotangent bundle action in another way that such terms do not appear. We derive the action (5.11) with the similar method used in subsection 4.1 . Using the isomorphism $T^{*} Q^{2} \simeq T^{*} \mathbf{C} P^{1} \times T^{*} \mathbf{C} P^{1}$, we can represent the $T^{*} Q^{2}$ action as a sum of two $T^{*} \mathbf{C} P^{1}$ action with different coordinate variables. Although naive summation is not written by geometrical quantities, similarly to the tangent bundle case, we can write the action in terms of metric and Riemann tensor. Finally we can reach the same form with (5.11) which is written in terms on $\xi$ and $\chi$ with base manifold coordinates. It is shown in Appendix C] Discussion on $n=3$ case is given in Appendix D.

\section{Discussion}

We would like to propose possible future works. Potential terms in $\mathcal{N}=2$ hyper-Kähler nonlinear sigma models are known to be written in the form of the square of a Killing vector of the target space [53, 54. Recently these potentials in the framework of the projective superspace has been established [55] by gauging the isometry to obtain the potential and then by freezing it. Using this method we could construct the potential of our $T^{(*)} Q^{n}$ nonlinear sigma model. Massive extension of $T^{*} \mathbf{C} P^{n}$ [56] and $T^{*}$ Grassmann [57] nonlinear sigma models were formulated by the hyper-Kähler quotient in the harmonic superspace formalism as well as components and $\mathcal{N}=1$ superfields. These models are known to admit various (composite) BPS solitons, like domain walls [58, 56, [59, 15], (Q-)lump-strings 60] (vortex-strings 61]), domain wall junctions [62], strings stretched between walls [63, 64, intersecting vortex-strings [65, 66], and other solitons 67. See [68, 69, 70] for a review in this subject. So constructing the massive $T^{*} Q^{n}$ model and investigating BPS solitons in it are interesting future directions.

We have seen in the Lagrangian (1.1) in the introduction that the $\mathcal{N}=1 Q^{n}$ model can be constructed by the Kähler quotient. It has been found [59, 15, 61, 62, 65, that the hyper-Kähler quotient construction is crucial in solving the BPS equations to construct all the exact soliton solutions. Therefore, hyper-Kähler quotient construction of the (massive) $T^{*} Q^{n}$ model is awaited. 
$\mathcal{N}=1$ superfield formalism is difficult to perform it because the superpotential exists even in the massless case (1.1). The projective superspace formalism should be useful to construct the massive $T^{*} Q^{n}$ model in hyper-Kähler quotient.

The cotangent bundle over the projective space $\mathbf{C} P^{n-1}$ can be locally written as

$$
T^{*} \mathbf{C} P^{n-1}=T^{*}\left[\frac{S U(n)}{S U(n-1) \times U(1)}\right] \simeq \mathbf{R} \times \frac{S U(n)}{S U(n-2)} .
$$

Therefore, this is in cohomogeneity one. It was proved [71 that this is the unique cohomogeneity one hyper-Kähler manifold. On the other hand, $T^{*} Q^{n-2}$ can be locally written as

$$
T^{*} Q^{n-2}=T^{*}\left[\frac{S O(n)}{S O(n-2) \times U(1)}\right] \simeq \mathbf{R}^{2} \times \frac{S O(n)}{S O(n-4)}
$$

which is in cohomogeneity two. Cohomogeneity two hyper-Kähler manifolds were discussed in 72 but complete classification is not yet known at least to our knowledge. We hope our example is useful to explore hyper-Kähler manifolds with higher cohomogeneity.

There exist other Hermitian symmetric spaces, $G_{n, m}=S U(n) /[S U(n-m) \times S U(m) \times U(1)]$, $S O(2 n) / U(n), S p(n) / U(n), E_{6} / S O(10) \times U(1)$ and $E_{7} / E_{6} \times U(1)$. A Kähler quotient construction of these manifolds was given in [48. ${ }^{8}$ It should be possible to construct (co)tangent bundles over Hermitian symmetric spaces [25] in the framework of the projective superspace. Extension to (co)tangent bundle over arbitrary homogeneous Kähler manifold $G / H$ is one goal of this subject. To this end, tangent bundles are in more compact form than cotangent bundles as seen in the cases of $\mathbf{C} P^{n}(3.19)$ and $Q^{n}$ (4.10). General form of the Kähler potential of tangent bundle over arbitrary Kähler manifold [26. 27] expanded in terms of a tangent vector $\Sigma$ was proposed in [43, 44]. There, each coefficient should be written in terms of the metric, curvature, covariant derivative like the cases of $\mathbf{C} P^{n}$ (3.19) and $Q^{n}$ (4.10), but explicit expression is not known in general. This expansion is very similar with the Kähler normal coordinate expansion [74]. We hope that Kähler normal coordinates are useful toward the construction of general action of hyper-Kähler nonlinear sigma models on (co)tangent bundle over arbitrary Kähler manifold.

\section{Acknowledgements}

We would like to thank Rikard von Unge for useful discussions and comments, especially, on duality between polar and $O(2)$ multiplets. We thank Kiyoshi Higashijima and Ulf Lindström for discussions. M. A. acknowledges the Institute of Physics in Prague and the Masaryk university for their hospitality while M. N. is grateful to the Helsinki Institute of Physics for their hospitality. The work of M. A. is supported by the bilateral program of Japan Society for the Promotion of Science and Academy of Finland, "Scientist Exchanges" while the work of M. N. is supported by Japan Society for the Promotion of Science under the Post-doctoral Research Program.

\footnotetext{
${ }^{8}$ The Calabi-Yau metrics on line bundles over Hermitian symmetric spaces were constructed [51, 73], which are all in cohomogeneity one.
} 


\section{Appendix}

\section{A Duality between polar and $O(2)$ multiplets}

Here we show the other way to eliminate infinite number of auxiliary fields, which is not mentioned in the text. The point is to perform a duality transformation [32, 75] between the polar multiplet $\Upsilon$ and real $O(2)$ multiplet defined by

$$
\eta=\bar{\Phi}+\zeta \Sigma-\zeta^{2} \Phi, \quad \Sigma=\bar{\Sigma} .
$$

The duality transformation is possible only if the polar multiplet appears as the linear combination such that $\Upsilon+\bar{\Upsilon}$ in an action. After the duality transformation, the action is described by $O(2)$ multiplet and there is thus no auxiliary fields. ${ }^{9}$ Let us illustrate this with a couple of examples.

Firstly we consider the flat space. Let us start with an action depending on the arctic projective multiplet $\Upsilon$ :

$$
S=\frac{1}{2} \int d^{8} z \oint \frac{d \zeta}{2 \pi i \zeta}(\Upsilon+\bar{\Upsilon})^{2}
$$

This is a sigma model in flat space since the Lagrangian consists of the Kähler potential $\Upsilon \bar{\Upsilon}$ of flat space and a Kähler transformation. Now we instead introduce an $O(2)$ multiplet $\eta$ and an unconstrained projective superfield $X$

$$
S=\int d^{8} z \oint \frac{d \zeta}{2 \pi i \zeta}\left(\frac{1}{2} X^{2}-X \frac{\eta}{\zeta}\right) .
$$

Varying with respect to $\eta$ tells us that $X$ can be written as a sum of an arctic and antarctic field $X=\Upsilon+\bar{\Upsilon}$ and we are back to the original model. If we on the other hand vary with respect to $X$ we get an equation

$$
X=\frac{\eta}{\zeta}
$$

Inserting this back into the action we get

$$
S=-\int d^{8} z \oint \frac{d \zeta}{2 \pi i \zeta} \frac{\eta^{2}}{\zeta^{2}},
$$

which is the action for a sigma model in flat space written with an $O(2)$ multiplet.

In the second example, we start from another Kähler potential in flat space:

$$
S=\int d^{8} z \oint \frac{d \zeta}{2 \pi i \zeta} e^{\Upsilon+\bar{\Upsilon}}
$$

\footnotetext{
${ }^{9}$ As more general case, there is duality between the real $O(2 p)$ and polar multiplets [36], which involves the case we will discuss below.
} 
In the same way, we introduce an $O(2)$ multiplet $\eta$ and a polar multiplet $X$ to get the action

$$
S=\int d^{8} z \oint \frac{d \zeta}{2 \pi i \zeta}\left(e^{X}-X \frac{\eta}{\zeta}\right)
$$

Again, integrating out $\eta$ tells us that $X$ can be written as a sum over an arctic and an antarctic multiplet while integrating out $X$ gives the action

$$
S=\int d^{8} z \oint \frac{d \zeta}{2 \pi i \zeta} \frac{\eta}{\zeta}\left(1-\ln \frac{\eta}{\zeta}\right)
$$

This is also a known form of the action for a sigma model in flat space. There is one unresolved issue here with the integration contour. Because of the nonsingle valuedeness of the log, the integration contour will not be simply a contour around the origin but rather a shaped curve around the zeroes of the quadratic polynomial $\eta(\zeta)$.

This method can be applied to any Kähler potential which can be written as a function of $\Upsilon+\bar{\Upsilon}$. Namely

$$
S=\int d^{8} z \oint \frac{d \zeta}{2 \pi i \zeta} f(\Upsilon+\bar{\Upsilon})=\int d^{8} z \oint \frac{d \zeta}{2 \pi i \zeta}\left(f(X)-X \frac{\eta}{\zeta}\right)
$$

Integrating out $X$ one gets

$$
f^{\prime}(X)=\frac{\eta}{\zeta}
$$

which can be formally inverted to give

$$
X=g\left(\frac{\eta}{\zeta}\right)
$$

Inserting this back into the action gives

$$
S=\int d^{8} z \oint \frac{d \zeta}{2 \pi i \zeta}\left\{f\left(g\left(\frac{\eta}{\zeta}\right)\right)-\frac{\eta}{\zeta} g\left(\frac{\eta}{\zeta}\right)\right\} .
$$

Technically we see that the new action is the Legendre transform of the old one. For example, starting with the Kähler potential of $T^{*} \mathbf{C} P^{1} \ln \left(1+e^{\Upsilon+} \bar{\Upsilon}\right)$, we get

$$
\begin{aligned}
S & =\int d^{8} z \oint \frac{d \zeta}{2 \pi i \zeta} \ln \left(1+e^{\Upsilon+\bar{\Upsilon}}\right) \\
& =\int d^{8} z \oint \frac{d \zeta}{2 \pi i \zeta}\left(-\frac{\eta}{\zeta} \ln \left(\frac{\eta}{\zeta}\right)-\left(1-\frac{\eta}{\zeta}\right) \ln \left(1-\frac{\eta}{\zeta}\right)\right)
\end{aligned}
$$

which is again a known form for the nonlinear sigma model with the Eguchi-Hanson metric. The contour is still an issue similarly to the flat case $e^{\Upsilon+\bar{\Upsilon}}$. As be seen, one sees how one does not have to solve the infinite number of equations although it is not applicable to the quadric surface. 


\section{B Deriving equations (5.7) and (5.8)}

In this Appendix, we show how to derive the equations (5.7) and (5.8). Substituting (5.2) and (5.3) into $\xi$ and $\chi$ leads to, after some algebra

$$
\begin{aligned}
& \xi=g^{i \bar{j}} \psi_{i} \bar{\psi}_{j} \\
& =\left(1-\frac{\rho}{r^{2}}+\frac{\rho^{2}}{2 r^{4}}+\frac{\sigma}{4 r^{2}}\right)^{-2} \\
& \times\left(\rho-\frac{2 \rho^{2}}{r^{2}}-\sigma+\frac{\rho^{3}}{r^{4}}+\frac{\rho \sigma}{r^{2}}+\frac{g^{i \bar{j}}}{4} R_{i \bar{l} p \bar{q}} R_{k \bar{j} m \bar{n}} \bar{U}^{\bar{l}} U^{p} \bar{U}^{\bar{q}} U^{k} U^{m} \bar{U}^{\bar{n}}\right) . \\
& \chi=R^{\bar{i} j \bar{l} k} \bar{\psi}_{i} \psi_{j} \bar{\psi}_{\bar{l}} \psi_{k} \\
& =\left(1-\frac{\rho}{r^{2}}+\frac{\rho^{2}}{2 r^{4}}+\frac{\sigma}{4 r^{2}}\right)^{-4} R^{\bar{j} i \bar{l} k} \\
& \times\left[g_{n \bar{j}} g_{i \bar{m}} g_{a \bar{l}} g_{k \bar{b}} U^{n} \bar{U}^{\bar{m}} U^{a} \bar{U}^{\bar{b}}\left(1-\frac{4 \rho}{r^{2}}+\frac{6 \rho^{2}}{r^{4}}-\frac{4 \rho^{3}}{r^{6}}+\frac{\rho^{4}}{r^{8}}\right)\right. \\
& +g_{n \bar{j}} g_{i \bar{m}} g_{k \bar{a}} R_{b \bar{l} c \bar{d}} U^{n} \bar{U}^{\bar{m}} \bar{U}^{\bar{a}} U^{b} U^{c} \bar{U}^{\bar{d}}\left(-2+\frac{6 \rho}{r^{2}}-\frac{6 \rho^{2}}{r^{4}}+\frac{2 \rho^{3}}{r^{6}}\right) \\
& +g_{i \bar{m}} g_{k \bar{a}} R_{n \bar{j} p \bar{q}} R_{b \bar{l} c \bar{d}} \bar{U}^{\bar{m}} U^{n} U^{p} \bar{U}^{\bar{q}} \bar{U}^{\bar{a}} U^{b} U^{c} \bar{U}^{\bar{d}}\left(\frac{3}{2}-\frac{3 \rho}{r^{2}}+\frac{3 \rho^{2}}{2 r^{4}}\right) \\
& +\frac{1}{2} g_{k \bar{x}} R_{i \bar{m} n \bar{p}} R_{a \bar{j} b \bar{c}} R_{y \bar{z} z \bar{w}} \bar{U}^{\bar{x}} \bar{U}^{\bar{m}} U^{n} \bar{U}^{\bar{p}} U^{a} U^{b} \bar{U}^{\bar{c}} U^{y} U^{z} \bar{U}^{\bar{w}}\left(-1+\frac{\rho}{r^{2}}\right) \\
& \left.+\frac{1}{16} R_{i \bar{m} n \bar{p}} R_{a \bar{j} b \bar{c}} R_{k \bar{x} y \bar{z}} R_{d \bar{l} e \bar{f}} \bar{U}^{\bar{m}} U^{n} \bar{U}^{\bar{p}} U^{a} U^{b} \bar{U}^{\bar{c}} \bar{U}^{\bar{x}} U^{y} \bar{U}^{\bar{z}} U^{d} U^{e} \bar{U}^{\bar{f}}\right] .
\end{aligned}
$$

It is seen that these equations are not closed with respect to $\rho$ and $\sigma$. However, using identities between metric and Riemann tensor, one can rewrite terms such like $g^{i \bar{j}} R_{i \bar{l} p \bar{q}} R_{k \bar{j} m \bar{n}} \bar{U}^{\bar{l}} U^{p} \bar{U}^{\bar{q}} U^{k} U^{m} \bar{U}^{\bar{n}}$ in the right hand side in (B.1) in terms of $\rho$ and $\sigma$.

First we consider Eq. (B.1). Calculating last term in the right hand side in (B.1) in the frame taken in (4.5), we find

$$
g^{i \bar{j}} R_{i \bar{l} p \bar{q}} R_{k \bar{j} m \bar{n}} \bar{U}^{\bar{l}} U^{p} \bar{U}^{\bar{q}} U^{k} U^{m} \bar{U}^{\bar{n}}=\frac{4\left|U^{1}\right|^{6}}{r^{4}\left(1+\frac{\left|\Phi_{1}\right|^{2}}{r^{2}}\right)^{6}}+\frac{4\left|U^{2}\right|^{6}}{r^{4}\left(1+\frac{\left|\Phi_{2}\right|^{2}}{r^{2}}\right)^{6}} .
$$

On the other hand, it is shown that

$$
\begin{aligned}
\rho \sigma= & -\frac{2\left|U^{1}\right|^{6}}{r^{2}\left(1+\frac{\left|\Phi_{1}\right|^{2}}{r^{2}}\right)^{6}}-\frac{2\left|U^{2}\right|^{6}}{r^{2}\left(1+\frac{\left|\Phi_{2}\right|^{2}}{r^{2}}\right)^{6}} \\
& -\frac{2}{r^{2}} G_{1} G_{2}\left|U^{1}\right|^{2}\left|U^{2}\right|^{2}\left(G_{1}\left|U^{1}\right|^{2}+G_{2}\left|U^{2}\right|^{2}\right) .
\end{aligned}
$$


Using ( $(\underline{\mathrm{B} .3}),(\underline{\mathrm{B} .4})$ and (4.9) with $\rho=g_{i \bar{j}} U^{i} \bar{U}^{\bar{j}}$, we get the following identity

$$
g^{i \bar{j}} R_{i \bar{l} \bar{p}} R_{k \bar{j} m \bar{n}} \bar{U}^{\bar{l}} U^{p} \bar{U}^{\bar{q}} U^{k} U^{m} \bar{U}^{\bar{n}}=-\frac{3 \rho \sigma}{r^{2}}-\frac{2}{r^{4}} \rho^{3} .
$$

Since this identify is written by geometric quantities, it holds in arbitrary frame. Substituting (B.5) into (B.1), we obtain (5.7).

Next we consider Eq. (B.2). Once again choosing the frame taken in (4.5), we find the following identities:

$$
\begin{aligned}
R^{\bar{i} j \bar{k} l} g_{n \bar{j}} g_{i \bar{m}} g_{i \bar{m}} g_{k \bar{a}} R_{n \bar{j} p \bar{q}} R_{b \bar{l} c \bar{d}} \bar{U}^{\bar{m}} U^{n} U^{p} \bar{U}^{\bar{q}} \bar{U}^{\bar{a}} U^{b} U^{c} \bar{U}^{\bar{d}} & =\frac{4 \rho^{4}}{r^{6}}-\frac{\sigma^{2}}{r^{2}}+\frac{4 \rho^{2} \sigma}{r^{4}},(\mathrm{~B} .6) \\
R^{\bar{i} j \bar{k} l} g_{n \bar{j}} g_{i \bar{m}} g_{k \bar{x}} R_{i \bar{m} n \bar{p}} R_{a \bar{j} b \bar{c}} R_{y \bar{l} z \bar{w}} \bar{U}^{\bar{x}} \bar{U}^{\bar{m}} U^{n} \bar{U}^{\bar{p}} U^{a} U^{b} \bar{U}^{\bar{c}} U^{y} U^{z} \bar{U}^{\bar{w}} & =\frac{5 \rho \sigma^{2}}{r^{4}}-\frac{4 \rho^{5}}{r^{8}}, \\
R^{\bar{i} j \bar{k} l} g_{n \bar{j}} g_{i \bar{m}} R_{i \bar{m} n \bar{p}} R_{a \bar{j} b \bar{c}} R_{k \bar{x} y \bar{z}} R_{d \bar{l} e \bar{f}} \bar{U}^{\bar{m}} U^{n} \bar{U}^{\bar{p}} U^{a} U^{b} \bar{U}^{\bar{c}} \bar{U}^{\bar{x}} U^{y} \bar{U}^{\bar{z}} U^{d} U^{e} \overline{U^{\bar{f}}} & =\frac{\sigma^{3}}{r^{4}}-\frac{12 \rho^{4} \sigma}{r^{8}}-\frac{12 \rho^{2} \sigma^{2}}{r^{6}} .
\end{aligned}
$$

Substituting (B.5) and (B.6) $-(\underline{B .8})$ into (B.2), we find the equation (5.8).

\section{Derivation of cotangent bundle without using Legendre transformation}

Instead of using the Legendre transformation, we can directly obtain the cotangent bundle action (5.11) for $T^{*} Q^{2}$ case. Similarly to the tangent bundle case, with the isomorphism $T^{*} Q^{2}=$ $T^{*} \mathbf{C} P^{1} \times T^{*} \mathbf{C} P^{1}$, the action for $T^{*} Q^{2}$ can be written as a direct sum of two actions of cotangent bundle over $\mathbf{C} P^{1}$. Using the action (3.23), we have

$$
\begin{aligned}
S= & \int d^{8} z\left\{r^{2} \ln \left(1+\frac{\left|\Phi^{i}\right|^{2}}{r^{2}}+\frac{\left|\Phi^{1}\right|^{2}\left|\Phi^{2}\right|^{2}}{r^{4}}\right)\right. \\
& \left.-r^{2} \ln f\left(\kappa_{1}\right)+2 r^{2} \frac{\kappa_{1}}{f\left(\kappa_{1}\right)}-r^{2} \ln f\left(\kappa_{2}\right)+2 r^{2} \frac{\kappa_{2}}{f\left(\kappa_{2}\right)}\right\},
\end{aligned}
$$

where

$$
f\left(\kappa_{i}\right)=\frac{1}{2}\left(1+\sqrt{1+4 \kappa_{i}}\right), \quad \kappa_{i}=\frac{1}{r^{2}} G_{i}^{-1} \psi_{i} \bar{\psi}_{\bar{i}}, \quad \text { (no sum). }
$$

Here $G_{i}$ is defined in (4.6). In the following, we rewrite the action (C.1) in terms of geometric quantities similarly to the tangent bundle case. We consider the two quantities $\xi=g^{i \bar{j}} \psi_{i} \bar{\psi}_{\bar{j}}$ and $\chi=R^{i j} \bar{k} l \bar{\psi}_{i} \psi_{j} \bar{\psi}_{\bar{k}} \psi_{l}$ as possible invariant forms written by $\psi_{i}$ and $\bar{\psi}_{\bar{j}}$ as the same with in section 5. In the frame taken in (4.5), one finds

$$
\begin{aligned}
\xi & =\frac{1}{G_{1}}\left|\psi_{1}\right|^{2}+\frac{1}{G_{2}}\left|\psi_{2}\right|^{2}, \\
\chi & =-\frac{2}{G_{1}^{2} r^{2}}\left|\psi_{1}\right|^{4}-\frac{2}{G_{2}^{2} r^{2}}\left|\psi_{2}\right|^{4} .
\end{aligned}
$$


Using (C.3) and (C.4) leads to

$$
\begin{aligned}
& \xi^{2}=\frac{\left|\psi_{1}\right|^{4}}{G_{1}^{2}}+\frac{\left|\psi_{2}\right|^{4}}{G_{2}^{2}}+ \frac{2}{G_{1} G_{2}}\left|\psi_{1}\right|^{2}\left|\psi_{2}\right|^{2}=-\frac{r^{2}}{2} R^{\bar{i} j \bar{k} l} \bar{\psi}_{\bar{i}} \psi_{j} \bar{\psi}_{\bar{k}} \psi_{l}+\frac{2}{G_{1} G_{2}}\left|\psi_{1}\right|^{2}\left|\psi_{2}\right|^{2}, \\
& \Leftrightarrow \frac{1}{r^{2} G_{1} G_{2}}\left|\psi_{1}\right|^{2}\left|\psi_{2}\right|^{2}=\frac{\xi^{2}}{2 r^{4}}+\frac{\chi}{4 r^{2}} .
\end{aligned}
$$

From (C.3), (C.4 and , we get two equations

$$
\begin{aligned}
\kappa_{1}+\kappa_{2} & =\frac{\xi}{r^{2}}, \\
\kappa_{1} \kappa_{2} & =\frac{\xi^{2}}{2 r^{4}}+\frac{\chi}{4 r^{2}} .
\end{aligned}
$$

Solving (C.6) and (C.7) with respect to $\kappa_{1}$ and $\kappa_{2}$, we obtain

$$
\left(\kappa_{1}, \kappa_{2}\right)=\left(\frac{\xi}{2 r^{2}}+\frac{1}{2} \sqrt{-\frac{\xi^{2}}{r^{4}}-\frac{\chi}{r^{2}}}, \frac{\xi}{2 r^{2}}-\frac{1}{2} \sqrt{-\frac{\xi^{2}}{r^{4}}-\frac{\chi}{r^{2}}}\right) .
$$

Substituting back this solution into the action (C.1) and performing the unitary transformation (4.2), we arrive at the action (5.11).

\section{D $T^{(*)} Q^{1}$ case}

Here we consider the $n=3$ case in the sigma model with $T^{(*)} Q^{n-2}$, say, $T^{(*)}[S O(3) / S O(2)] \simeq$ $T^{(*)} \mathbf{C} P^{1}$. In this case, everything is drastically simplified. The Kähler potential (1.8) in this case reduces to the $\mathbf{C} P^{1}$ 's one:

$$
K\left(\Phi^{i}, \bar{\Phi}_{j}\right)=\ln \left(1+\frac{|\Phi|^{2}}{r^{2}}+\frac{(\Phi)^{2}(\bar{\Phi})^{2}}{4 r^{4}}\right)=2 \ln \left(1+\frac{|\Phi|^{2}}{2 r^{2}}\right) .
$$

Eqs. (4.15)-(4.17) become

$$
\begin{aligned}
\lambda & =\epsilon \bar{\epsilon} \\
\beta_{+} & =0, \quad \beta_{-}=\sqrt{2 \epsilon \bar{\epsilon}} \\
\gamma_{+} & =2 \epsilon \lambda, \quad \gamma_{-}=0 .
\end{aligned}
$$

Now the transformation factor $V_{Q}$ in Eq. (4.19) can be simply written as

$$
\Sigma=V_{Q} \Sigma_{0}=\left(1+\frac{|\Phi|^{2}}{2 r^{2}}\right) \Sigma_{0}
$$

Substituting these expressions into (4.26), we find

$$
\Upsilon=\frac{\left(1+\frac{|\Phi|^{2}}{2 r^{2}}\right) \Phi+\zeta \Sigma}{1+\frac{|\Phi|^{2}}{2 r^{2}}-\frac{\zeta}{2 r^{2}} \bar{\Phi} \Sigma}
$$


This result coincides with one of (3.15) in the $n=2$ case with rescaling $r \rightarrow r / \sqrt{2}$.

We can also see that the actions (4.10) and (5.11) reduce to ones of $T \mathbf{C} P^{1}$ and $T^{*} \mathbf{C} P^{1}$ in the $n=3$ case. In this case, the covariant quantities $\rho, \sigma, \xi$ and $\chi$ become

$$
\begin{gathered}
\rho \rightarrow G|\Sigma|^{2}, \quad \sigma \rightarrow-\frac{1}{r^{2}} G^{2}|\Sigma|^{4}, \\
\xi \rightarrow G^{-1}|\psi|^{2}, \quad \chi \rightarrow-\frac{1}{r^{2}} G^{-2}|\psi|^{4},
\end{gathered}
$$

and Eqs. (C.8) and $f\left(\kappa_{i}\right)$ are

$$
\begin{gathered}
\kappa_{1}, \kappa_{2} \rightarrow \frac{|\psi|^{2}}{2 r^{2} G}, \\
f\left(\kappa_{1}\right), f\left(\kappa_{2}\right) \rightarrow f(\kappa),
\end{gathered}
$$

where $G=\left(1+|\Phi|^{2} /\left(2 r^{2}\right)\right)^{-1}$ and $f(\kappa)=\frac{1}{2}\left(1+\sqrt{1+\frac{2|\psi|^{2}}{r^{2} G}}\right)$. Using these formulas, we find that the actions for tangent and cotangent bundles coincide with (3.19) and (3.23) in the $n=2$ case, respectively, with rescaling $r \rightarrow r / \sqrt{2}$.

\section{References}

[1] M. F. Atiyah, N. J. Hitchin, V. G. Drinfeld and Y. I. Manin, "Construction Of Instantons," Phys. Lett. A 65 (1978) 185.

[2] W. Nahm, "A Simple Formalism For The BPS Monopole," Phys. Lett. B 90 (1980) 413.

[3] M. F. Atiyah and N. J. Hitchin, "Low-Energy Scattering Of Nonabelian Monopoles," Phys. Lett. A 107 (1985) 21; "Low-Energy Scattering Of Nonabelian Magnetic Monopoles," Phil. Trans. Roy. Soc. Lond. A 315 (1985) 459.

[4] T. Eguchi and A. J. Hanson, "Asymptotically Flat Selfdual Solutions To Euclidean Gravity," Phys. Lett. B 74 (1978) 249; T. Eguchi, P. B. Gilkey and A. J. Hanson, "Gravitation, Gauge Theories And Differential Geometry," Phys. Rept. 66 (1980) 213; G. W. Gibbons and S. W. Hawking, "Gravitational Multi - Instantons," Phys. Lett. B 78 (1978) 430; N. J. Hitchin, "Polygons and gravitons," in *Gibbons, G.W. (ed.), Hawking, S.W. (ed.): Euclidean quantum gravity* 527-538.

[5] P. B. Kronheimer, "The Construction Of ALE Spaces As Hyperkähler Quotients," J. Diff. Geom. 29 (1989) 665.

[6] P. B. Kronheimer and H. Nakajima, "Yang-Mills instantons on ALE gravitational instantons", Math. Ann. 288 (1990) 263; H. Nakajima, "Instantons on ALE spaces, quiver varieties, and Kac-Moody algebras", Duke Math. J. 76 (1994) 365.

[7] L. Alvarez-Gaume and D. Z. Freedman, "Geometrical Structure And Ultraviolet Finiteness In The Supersymmetric Sigma Model," Commun. Math. Phys. 80 (1981) 443. 
[8] U. Lindström and M. Roček, "Scalar Tensor Duality And N=1, N=2 Nonlinear Sigma Models," Nucl. Phys. B 222 (1983) 285.

[9] N. J. Hitchin, A. Karlhede, U. Lindström and M. Roček, "Hyperkähler Metrics And Supersymmetry," Commun. Math. Phys. 108 (1987) 535.

[10] P. C. Argyres, M. R. Plesser and N. Seiberg, "The Moduli Space of N=2 SUSY QCD and Duality in N=1 SUSY QCD," Nucl. Phys. B 471 (1996) 159 arXiv:hep-th/9603042.

[11] I. Antoniadis and B. Pioline, "Higgs branch, hyperKähler quotient and duality in SUSY N = 2 Yang-Mills theories," Int. J. Mod. Phys. A 12 (1997) 4907 arXiv:hep-th/9607058.

[12] H. Pedersen and S. P. Yat, "Hyperkähler Metrics And A Generalization Of The Bogomolny Equations," Commun. Math. Phys. 117 (1988) 569.

[13] G. W. Gibbons, R. Goto and P. Rychenkova, "HyperKähler quotient construction of BPS monopole moduli spaces," Commun. Math. Phys. 186 (1997) 585 arXiv:hep-th/9608085.

[14] R. Bielawski, "Complete hyperKähler 4n-manifolds with $n$ commuting tri-Hamiltonian vector fields," Math. Ann. 314 (1999) 505 arXiv:math.dg/9808134.

[15] M. Eto, Y. Isozumi, M. Nitta, K. Ohashi, K. Ohta, N. Sakai and Y. Tachikawa, "Global structure of moduli space for BPS walls," Phys. Rev. D 71 (2005) 105009 arXiv:hep-th/0503033.

[16] U. Lindström, M. Roček and R. von Unge, "Hyperkähler quotients and algebraic curves," JHEP 0001 (2000) 022 arXiv:hep-th/9908082.

[17] A. Borel, "Kählerian coset spaces of semisimple Lie groups," Proc. Natl. Acad. Sci. 40 (1954) 1147.

[18] M. Bordemann, M. Forger and H. Romer, "Homogeneous Kähler Manifolds: Paving The Way Towards New Supersymmetric Sigma Models," Commun. Math. Phys. 102 (1986) 605 .

[19] K. Itoh, T. Kugo and H. Kunitomo, "Supersymmetric Nonlinear Realization For Arbitrary Kählerian Coset Space G/H," Nucl. Phys. B 263 (1986) 295.

[20] Arthur L. Besse, " Einstein manifolds," Springer-Verlag, Berlin, 1987.

[21] P. B. Kronheimer, "A hyper-Kählerian structure on coadjoint orbits of a semisimple complex group," J. London Math. Soc. (2) 42 (1990) 193.

[22] M. B. Stenzel, "Ricci-flat metrics on the complexification of a compact rank one symmetric space," Manuscripta Math. 80 (1993) 151.

[23] M. Cvetič, G. W. Gibbons, H. Lu and C. N. Pope, "Ricci-flat metrics, harmonic forms and brane resolutions," Commun. Math. Phys. 232 (2003) 457 arXiv:hep-th/0012011. 
[24] K. Higashijima, T. Kimura and M. Nitta, "Supersymmetric nonlinear sigma models on Ricci-flat Kähler manifolds with O(N) symmetry," Phys. Lett. B 515 (2001) 421 arXiv:hep-th/0104184.

[25] O. Biquard and P. Gauduchon, "Hyper-Kähler metrics on cotangent bundles of Hermitian symmetric spaces," Geometry and physics (Aarhus, 1995) 287-298, Lecture Notes in Pure and Appl. Math., 184, Dekker, New York, 1997; "La métrique hyperkählérienne des orbites coadjointes de type symétrique d'un groupe de Lie complexe semi-simple [The hyper-Kähler metric of coadjoint orbits of symmetric type of a complex semisimple Lie group]," C. R. Acad. Sci. Paris Sér. I Math. 323 (1996) 1259-1264.

[26] D. Kaledin, "A canonical hyperkähler metric on the total space of a cotangent bundle," Quaternionic structures in mathematics and physics (Rome, 1999), 195-230, Univ. Studi Roma "La Sapienza", Rome, 1999 arXiv:alg-geom/9710026.

[27] B. Feix, "Hyperkähler metrics on cotangent bundles," J. Reine Angew. Math. 532 (2001) $33-46$.

[28] A. Galperin, E. Ivanov, S. Kalitsyn, V. Ogievetsky and E. Sokatchev, "Unconstrained N=2 Matter, Yang-Mills And Supergravity Theories In Harmonic Superspace," Class. Quant. Grav. 1 (1984) 469.

[29] A. S. Galperin, E. A. Ivanov, V. I. Ogievetsky and E. S. Sokatchev, "Harmonic Superspace," (Cambridge Monographs on Mathematical Physics) Cambridge University Press (2001).

[30] A. Karlhede, U. Lindström and M. Roček, "Selfinteracting Tensor Multiplets In N=2 Superspace," Phys. Lett. B 147 (1984) 297.

[31] S. V. Ketov, B. B. Lokhvitsky and I. V. Tyutin, "Hyperkähler Sigma Models In Extended Superspace," Theor. Math. Phys. 71 (1987) 496 [Teor. Mat. Fiz. 71 (1987) 226].

[32] U. Lindström and M. Roček, "New Hyperkähler Metrics And New Supermultiplets," Commun. Math. Phys. 115 (1988) 21.

[33] U. Lindström and M. Roček, "N=2 Superyang-Mills Theory In Projective Superspace," Commun. Math. Phys. 128 (1990) 191.

[34] S. V. Ketov, "New N=2 Matter Coupling In Superspace," Int. J. Mod. Phys. A 3 (1988) 703.

[35] S. V. Ketov, "Quantum Non-linear Sigma-Models : From Quantum Field Theory to Supersymmetry, Conformal Field Theory, Black Holes and Strings," Springer (2000).

[36] F. Gonzalez-Rey, M. Roček, S. Wiles, U. Lindström and R. von Unge, "Feynman rules in $\mathrm{N}=2$ projective superspace. I: Massless hypermultiplets," Nucl. Phys. B 516 (1998) 426 arXiv:hep-th/9710250. 
[37] F. Gonzalez-Rey and R. von Unge, "Feynman rules in N $=2$ projective superspace. II: Massive hypermultiplets," Nucl. Phys. B 516 (1998) 449 arXiv:hep-th/9711135; F. Gonzalez-Rey, "Feynman rules in $\mathrm{N}=2$ projective superspace. III: Yang-Mills multiplet," arXiv:hep-th/9712128.

[38] S. M. Kuzenko, "Projective superspace as a double-punctured harmonic superspace," Int. J. Mod. Phys. A 14 (1999) 1737 arXiv:hep-th/9806147.

[39] S. M. Kuzenko and W. D. Linch, "On five-dimensional superspaces," arXiv:hep-th/0507176.

[40] S. J. Gates, S. Penati and G. Tartaglino-Mazzucchelli, "6D supersymmetry, projective superspace and 4D, N = 1 superfields," arXiv:hep-th/0508187.

[41] S. M. Kuzenko, "On compactified harmonic/projective superspace, 5D superconformal theories, and all that," arXiv:hep-th/0601177.

[42] J. Grundberg and U. Lindström, "Actions For Linear Multiplets In Six-Dimensions," Class. Quant. Grav. 2 (1985) L33.

[43] S. J. Gates and S. M. Kuzenko, "The CNM-hypermultiplet nexus," Nucl. Phys. B 543 (1999) 122 arXiv:hep-th/9810137.

[44] S. J. Gates and S. M. Kuzenko, "4D N $=2$ supersymmetric off-shell sigma models on the cotangent bundles of Kähler manifolds," Fortsch. Phys. 48 (2000) 115 arXiv:hep-th/9903013.

[45] S. J. Gates, T. Hubsch and S. M. Kuzenko, "CNM models, holomorphic functions and projective superspace C-maps," Nucl. Phys. B 557 (1999) 443 arXiv:hep-th/9902211.

[46] S. J. Gates, M. T. Griaru, M. Roček and W. Siegel, "Superspace, Or One Thousand And One Lessons In Supersymmetry," Front. Phys. 58 (1983) 1 arXiv:hep-th/0108200.

[47] T. L. Curtright and D. Z. Freedman, "Nonlinear Sigma Models With Extended Supersymmetry In Four-Dimensions," Phys. Lett. B 90 (1980) 71 [Erratum-ibid. B 91 (1980) 487]; L. Alvarez-Gaume and D. Z. Freedman, "Ricci Flat Kahler Manifolds And Supersymmetry," Phys. Lett. B 94 (1980) 171; M. Rocek and P. K. Townsend, "Three Loop Finiteness Of The N=4 Supersymmetric Nonlinear Sigma Model," Phys. Lett. B 96 (1980) 72.

[48] K. Higashijima and M. Nitta, "Supersymmetric nonlinear sigma models as gauge theories," Prog. Theor. Phys. 103 (2000) 635 arXiv:hep-th/9911139.

[49] K. Higashijima, T. Kimura, M. Nitta and M. Tsuzuki, "Large-N limit of N = 2 supersymmetric $Q^{N}$ model in two dimensions," Prog. Theor. Phys. 105 (2001) 261 arXiv:hep-th/0010272.

[50] F. Delduc and G. Valent, "Classical And Quantum Structure Of The Compact Kählerian Sigma Models," Nucl. Phys. B 253 (1985) 494; U. Ellwanger, "Supersymmetric Sigma Models In Four-Dimensions As Quantum Theories," Nucl. Phys. B 281 (1987) 489. 
[51] K. Higashijima, T. Kimura and M. Nitta, "A note on conifolds," Phys. Lett. B 518 (2001) 301 arXiv:hep-th/0107100.

[52] A. M. Perelomov, "Chiral Models: Geometrical Aspects," Phys. Rept. 146 (1987) 135.

[53] L. Alvarez-Gaume and D. Z. Freedman, "Potentials For The Supersymmetric Nonlinear Sigma Model," Commun. Math. Phys. 91 (1983) 87.

[54] J. Bagger and C. Xiong, " $\mathrm{N}=2$ nonlinear sigma models in $\mathrm{N}=1$ superspace: Four and five dimensions," arXiv:hep-th/0601165.

[55] S. M. Kuzenko, "On superpotentials for nonlinear sigma-models with eight supercharges," arXiv:hep-th/0602050.

[56] M. Arai, M. Naganuma, M. Nitta and N. Sakai, "Manifest supersymmetry for BPS walls in N = 2 nonlinear sigma models," Nucl. Phys. B 652 (2003) 35 arXiv:hep-th/0211103; "BPS wall in $\mathrm{N}=2$ SUSY nonlinear sigma model with Eguchi-Hanson manifold," in Garden of Quanta - In honor of Hiroshi Ezawa, Eds. by J. Arafune et al. (World Scientific Publishing Co. Pte. Ltd. Singapore, 2003) pp 299-325, arXiv:hep-th/0302028.

[57] M. Arai, M. Nitta and N. Sakai, "Vacua of massive hyper-Kähler sigma models of nonAbelian quotient," Prog. Theor. Phys. 113 (2005) 657 arXiv:hep-th/0307274 ; "Massive Hyper-Kähler sigma models and BPS domain walls," Phys. Atom. Nucl. 68 (2005) 1634 [Yad. Fiz. 68 (2005) 1698] arXiv:hep-th/0401102.

[58] E. R. C. Abraham and P. K. Townsend, "Q kinks," Phys. Lett. B 291 (1992) 85; "More on Q kinks: A (1+1)-dimensional analog of dyons," Phys. Lett. B 295 (1992) 225; J. P. Gauntlett, D. Tong and P. K. Townsend, "Multi-domain walls in massive supersymmetric sigma-models," Phys. Rev. D 64 (2001) 025010 arXiv:hep-th/0012178; D. Tong, "The moduli space of BPS domain walls," Phys. Rev. D 66 (2002) 025013 arXiv:hep-th/0202012.

[59] Y. Isozumi, M. Nitta, K. Ohashi and N. Sakai, "Construction of non-Abelian walls and their complete moduli space," Phys. Rev. Lett. 93 (2004) 161601 arXiv:hep-th/0404198; "Non-Abelian walls in supersymmetric gauge theories," Phys. Rev. D 70 (2004) 125014 arXiv:hep-th/0405194; M. Eto, Y. Isozumi, M. Nitta, K. Ohashi, K. Ohta and N. Sakai, "D-brane construction for non-Abelian walls," Phys. Rev. D 71 (2005) 125006 arXiv:hep-th/0412024.

[60] R. S. Ward, "Slowly Moving Lumps In The C $P^{1}$ Model In (2+1)-Dimensions," Phys. Lett. B 158 (1985) 424; I. Stokoe and W. J. Zakrzewski, "Dynamics Of Solutions Of The CP And C $P^{2}$ Models In (2+1)-Dimensions," Z. Phys. C 34 (1987) 491; R. A. Leese, "Q lumps and their interactions," Nucl. Phys. B 366 (1991) 283; E. Abraham, "Nonlinear sigma models and their Q lump solutions," Phys. Lett. B 278 (1992) 291. 
[61] M. Eto, Y. Isozumi, M. Nitta, K. Ohashi and N. Sakai, "Moduli space of non-Abelian vortices," arXiv:hep-th/0511088, M. Eto, T. Fujimori, Y. Isozumi, M. Nitta, K. Ohashi, K. Ohta and N. Sakai, "Non-Abelian vortices on cylinder: Duality between vortices and walls," arXiv:hep-th/0601181.

[62] M. Eto, Y. Isozumi, M. Nitta, K. Ohashi and N. Sakai, "Webs of walls," Phys. Rev. D 72 (2005) 085004 arXiv:hep-th/0506135; "Non-abelian webs of walls," Phys. Lett. B 632 (2006) 384 arXiv:hep-th/0508241]; M. Eto, Y. Isozumi, M. Nitta, K. Ohashi, K. Ohta and N. Sakai, "D-brane configurations for domain walls and their webs," AIP Conf. Proc. 805 (2005) 354 arXiv:hep-th/0509127.

[63] Y. Isozumi, M. Nitta, K. Ohashi and N. Sakai, "All exact solutions of a 1/4 Bogomol'nyiPrasad-Sommerfield equation," Phys. Rev. D 71 (2005) 065018 arXiv:hep-th/0405129.

[64] J. P. Gauntlett, R. Portugues, D. Tong and P. K. Townsend, "D-brane solitons in supersymmetric sigma-models," Phys. Rev. D 63 (2001) 085002 arXiv:hep-th/0008221; N. Sakai and D. Tong, "Monopoles, vortices, domain walls and D-branes: The rules of interaction," JHEP 0503 (2005) 019 arXiv:hep-th/0501207.

[65] M. Eto, Y. Isozumi, M. Nitta, K. Ohashi and N. Sakai, "Instantons in the Higgs phase," Phys. Rev. D 72 (2005) 025011 arXiv:hep-th/0412048.

[66] M. Naganuma, M. Nitta and N. Sakai, "BPS lumps and their intersections in N = 2 SUSY nonlinear sigma models," Grav. Cosmol. 8 (2002) 129 arXiv:hep-th/0108133; R. Portugues and P. K. Townsend, "Sigma-model soliton intersections from exceptional calibrations," JHEP 0204 (2002) 039 arXiv:hep-th/0203181.

[67] M. Eto, Y. Isozumi, M. Nitta and K. Ohashi, "1/2, 1/4 and 1/8 BPS equations in SUSY Yang-Mills-Higgs systems: Field theoretical brane configurations," arXiv:hep-th/0506257; M. Eto, M. Nitta, K. Ohashi and D. Tong, "Skyrmions from instantons inside domain walls," Phys. Rev. Lett. 95 (2005) 252003 arXiv:hep-th/0508130; J. P. Gauntlett, D. Tong and P. K. Townsend, "Supersymmetric intersecting domain walls in massive hyper-Kähler sigma models," Phys. Rev. D 63 (2001) 085001 arXiv:hep-th/0007124.

[68] D. Tong, "TASI lectures on solitons," arXiv:hep-th/0509216.

[69] M. Eto, Y. Isozumi, M. Nitta, K. Ohashi and N. Sakai, "Solitons in the Higgs phase - the moduli matrix approach -," arXiv:hep-th/0602170.

[70] Y. Isozumi, M. Nitta, K. Ohashi and N. Sakai, "Walls and vortices in supersymmetric non-Abelian gauge theories," to appear in the proceedings of "NathFest" at PASCOS conference, Northeastern University, Boston, Ma, August 2004 arXiv:hep-th/0410150; "NonAbelian walls and vortices in supersymmetric theories," Proceedings of 12th International Conference on Supersymmetry and Unification of Fundamental Interactions (SUSY 04), Tsukuba, Japan, 17-23 Jun 2004, edited by K. Hagiwara et al. (KEK, 2004) p.1 - p.16 arXiv:hep-th/0409110; M. Eto, Y. Isozumi, M. Nitta, K. Ohashi and N. Sakai, AIP Conf. Proc. 805, 266 (2005) arXiv:hep-th/0508017. 
[71] A. Dancer and A. Swann, "Hyper-Kähler metrics of cohomogeneity one," J. Geom. Phys. 21 (1997) 218-230.

[72] A. Swann and P. Kobak, "HyperKähler potentials in cohomogeneity two," J. Reine Angew. Math. 531 (2001) 121-139 arXiv:math.DG/0001024.

[73] K. Higashijima, T. Kimura and M. Nitta, "Ricci-flat Kähler manifolds from supersymmetric gauge theories," Nucl. Phys. B 623 (2002) 133 arXiv:hep-th/0108084; "Gauge theoretical construction of non-compact Calabi-Yau manifolds," Annals Phys. 296 (2002) 347 arXiv:hep-th/0110216; "Calabi-Yau manifolds of cohomogeneity one as complex line bundles," Nucl. Phys. B 645 (2002) 438 arXiv:hep-th/0202064; "Construction of supersymmetric nonlinear sigma models on noncompact Calabi-Yau manifolds with isometry," Nucl. Phys. Proc. Suppl. 117 (2003) 867 arXiv:hep-th/0210034.

[74] K. Higashijima and M. Nitta, "Kähler normal coordinate expansion in supersymmetric theories," Prog. Theor. Phys. 105 (2001) 243 arXiv:hep-th/0006027; K. Higashijima, E. Itou and M. Nitta, "Normal coordinates in Kähler manifolds and the background field method," Prog. Theor. Phys. 108 (2002) 185 arXiv:hep-th/0203081.

[75] B. de Wit, M. Roček and S. Vandoren, "Hypermultiplets, hyperkähler cones and quaternion-Kähler geometry," JHEP 0102 (2001) 039 arXiv:hep-th/0101161. 